\title{
Article \\ Ileum Gene Expression in Response to Acute Systemic Inflammation in Mice Chronically Fed Ethanol: Beneficial Effects of Elevated Tissue n-3 PUFAs
}

\author{
Josiah E. Hardesty ${ }^{1,2,+}$, Jeffrey B. Warner ${ }^{1,2,+}$, Ying L. Song ${ }^{1}$, Eric C. Rouchka ${ }^{3}$, Craig J. McClain ${ }^{1,2,4,5,6} \mathbb{D}^{\text {, }}$ \\ Dennis R. Warner ${ }^{1}$ and Irina A. Kirpich ${ }^{1,2,4,5, *}$
}

1 Division of Gastroenterology, Hepatology, and Nutrition, Department of Medicine, University of Louisville, Louisville, KY 40202, USA; josiah.hardesty@louisville.edu (J.E.H.); jeffrey.warner.1@louisville.edu (J.B.W.); ying.song@louisville.edu (Y.L.S.); craig.mcclain@louisville.edu (C.J.M.); dennis.warner@louisville.edu (D.R.W.)

2 Department of Pharmacology and Toxicology, School of Medicine, University of Louisville, Louisville, KY 40202, USA

3 Department of Computer Science and Engineering, Speed School of Engineering, University of Louisville, Louisville, KY 40292, USA; eric.rouchka@louisville.edu

4 University of Louisville Alcohol Center, School of Medicine, University of Louisville, Louisville, KY 40292, USA

5 University of Louisville Hepatology and Toxicology Center, School of Medicine, University of Louisville, Louisville, KY 40292, USA

6 Robley Rex Veterans Medical Center, Louisville, KY 40206, USA

* Correspondence: irina.kirpich@louisville.edu

$\dagger$ These authors contributed equally to this work.

Citation: Hardesty, J.E.; Warner, J.B.; Song, Y.L.; Rouchka, E.C.; McClain,

C.J.; Warner, D.R.; Kirpich, I.A. Ileum

Gene Expression in Response to

Acute Systemic Inflammation in Mice Chronically Fed Ethanol: Beneficial Effects of Elevated Tissue n-3 PUFAs. Int. J. Mol. Sci. 2021, 22, 1582. https://doi.org/10.3390/ijms 22041582

Academic Editor: Gabriella Calviello Received: 30 December 2020

Accepted: 1 February 2021

Published: 4 February 2021

Publisher's Note: MDPI stays neutral with regard to jurisdictional claims in published maps and institutional affiliations.

Copyright: (c) 2021 by the authors. Licensee MDPI, Basel, Switzerland. This article is an open access article distributed under the terms and conditions of the Creative Commons Attribution (CC BY) license (https:// creativecommons.org/licenses/by/ $4.0 /)$.

\begin{abstract}
Chronic alcohol consumption leads to disturbances in intestinal function which can be exacerbated by inflammation and modulated by different factors, e.g., polyunsaturated fatty acids (PUFAs). The mechanisms underlying these alterations are not well understood. In this study, RNA-seq analysis was performed on ileum tissue from WT and fat-1 transgenic mice (which have elevated endogenous n-3 PUFAs). Mice were chronically fed ethanol (EtOH) and challenged with a single lipopolysaccharide (LPS) dose to induce acute systemic inflammation. Both WT and fat-1 mice exhibited significant ileum transcriptome changes following EtOH + LPS treatment. Compared to WT, fat-1 mice had upregulated expression of genes associated with cell cycle and xenobiotic metabolism, while the expression of pro-inflammatory cytokines and pro-fibrotic genes was decreased. In response to EtOH + LPS, fat-1 mice had an increased expression of genes related to antibacterial B cells (APRIL and $\operatorname{IgA}$ ), as well as an elevation in markers of pro-restorative macrophages and $\gamma \delta \mathrm{T}$ cells that was not observed in WT mice. Our study significantly expands the knowledge of regulatory mechanisms underlying intestinal alterations due to $\mathrm{EtOH}$ consumption and inflammation and identifies the beneficial transcriptional effects of n-3 PUFAs, which may serve as a viable nutritional intervention for intestinal damage resulting from excessive alcohol consumption.
\end{abstract}

Keywords: alcohol; acute systemic inflammation; polyunsaturated fatty acids; intestine; transcriptome

\section{Introduction}

The intestinal epithelium serves several important functions including maintenance of the gut barrier and segregation of host tissue from bacteria and bacterial products [1] To this end, the intestinal epithelium undergoes continuous breakdown and restoration, a process which may be beneficially or detrimentally affected by external or internal factors, such as dietary fatty acids and inflammation [1,2]. For example, n-3 polyunsaturated fatty acid (PUFA) supplementation has beneficial effects with regard to intestinal health and function and the gut microbiome in humans [2,3]. In animals, n-3 PUFA supplementation improved intestinal tight junction integrity and reduced inflammation in a colitis model [4]. 
Similarly, transgenic mice that endogenously produce n-3 PUFAs from n6 PUFAs (fat-1 mice [5]) have reduced intestinal permeability when fed a hypercaloric diet [6] and are also protected from colitis [7]. Whereas n-3 PUFAs exert positive effects on intestinal health and function, dietary n-6 PUFAs (specifically in combination with chronic ethanol [EtOH] consumption) can disrupt intestinal barrier integrity, alter the gut microbiota [8-10], and lead to intestinal inflammation and abnormal gut barrier defense [11]. A compromised gut barrier allows bacteria and bacterial products such as lipopolysaccharide (LPS) from gram-negative bacteria to translocate to the blood and other organs [12], causing systemic inflammation and further exacerbating gut permeability and intestinal injury [13]. In addition, chronic intestinal inflammation can also promote intestinal fibrosis, leading to additional loss of gut barrier function [14]. We recently reported that fat-1 mice had attenuated $\mathrm{EtOH}$-induced alterations in intestinal homeostasis [15] that were associated with a markedly plastic transcriptome response to $\mathrm{EtOH}$ as well as specific transcriptional signatures [16]. Cell death, inflammation, and tuft cell markers were downregulated in fat-1 mice in response to $\mathrm{EtOH}$, while defense responses and PPAR signaling were upregulated [16]. Importantly, however, the molecular mechanisms underlying EtOH and $\mathrm{EtOH}+$ LPS-mediated intestinal pathology remain poorly understood.

Since EtOH consumption and acute systemic inflammation contribute to gut pathology, we aimed to elucidate the associated transcriptional responses using an unbiased RNA-seq based approach. Previously, we demonstrated improved intestinal health with n-3 PUFA enrichment in fat-1 transgenic mice (the mice that endogenously convert n6 to n3 PUFAs [15]) and identified the associated ileum transcriptional responses to $\mathrm{EtOH}$ [16]. The current study builds upon our previous work and aims to identify n-3 PUFA-regulated ileum transcriptional responses to acute systemic inflammation in mice chronically fed EtOH.

\section{Results}

2.1. Chronic EtOH Consumption Followed by LPS Challenge Leads to Global Ileum Gene Changes in Both WT and fat-1 Mice

To determine the effect of chronic EtOH consumption and LPS challenge on ileal gene expression, WT and fat-1 mice were fed an EtOH-containing diet with or without a single LPS challenge followed by ileum RNA-seq analysis (Figure 1A). There were 2276 differentially expressed genes (DEGs) in the ileum between WT EtOH+LPS vs. WT EtOH mice, 1583 DEGs between fat-1 EtOH + LPS vs. fat-1 EtOH mice, and 743 DEGs between fat-1 EtOH + LPS vs. WT EtOH + LPS (Figure 1B). There were 734 genes increased and 794 decreased exclusively in WT mice, and 435 genes increased and 403 decreased exclusively in fat 1 mice in response to EtOH + LPS vs. EtOH. There were commonly altered genes by LPS ( 445 genes increased and 303 decreased) in both WT and fat- 1 EtOH-fed mice (Figure 1C). A Venn diagram of the number of significant genes between fat-1 EtOH + LPS vs. fat-1 EtOH, WT EtOH + LPS vs. WT EtOH, and fat-1 EtOH + LPS is included as Figure S1. The most upregulated genes in the WT EtOH + LPS vs. WT EtOH comparison included Lcn2, Saa3, S100a8, Fabp1, and G6pc (568.8-, 166.6-, 140.1-, 88.3-, and 80.8-fold, respectively), while the downregulated genes included Cyp2c55, Pla2g4c, Gsdmc4, Gsdmc2, and Car1 $(-215-,-155.7-,-149.9-,-141.2-$, and -84.4-fold, respectively) (Table S1 and Figure 1D). The most upregulated genes in the fat- $1 \mathrm{EtOH}+\mathrm{LPS}$ vs. fat-1 EtOH comparison included Lcn2, Lct, Gata4, Gimd1, and Saa3 (496.8-, 402-, 134-, 123.9-, and 122.2-fold, respectively), while the downregulated genes included Cyp2c55, Cypd34, Gsdmc2, Hao2, and Car1 (-197.9-, -143.9-, $-127.1-,-122.3-$, and -99.1-fold, respectively) (Table S2 and Figure 1E). Gene Ontology (GO) processes increased in EtOH + LPS vs. EtOH-treated WT mice included response to stress, defense response, and inflammatory processes. In contrast, diminished GO processes included lipid metabolism, muscle contraction, and response to hormones (Figure 1F). GO processes enriched in fat-1 mice in response to EtOH + LPS vs. EtOH included immune system response and defense responses. Diminished GO processes included transport, development, and muscle contraction (Figure 1G). 
A

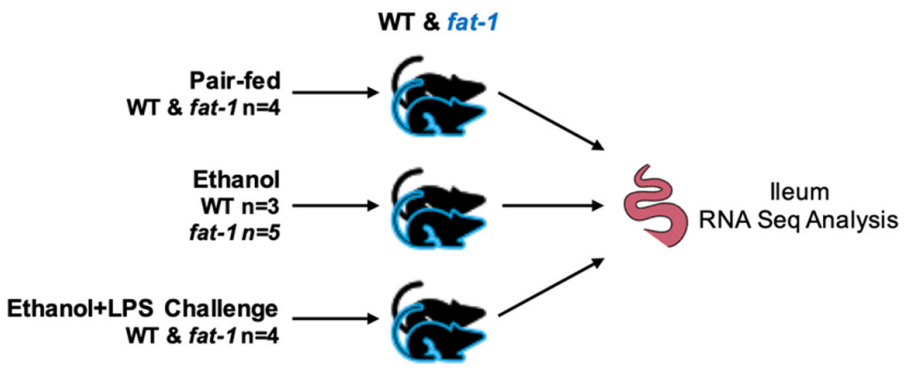

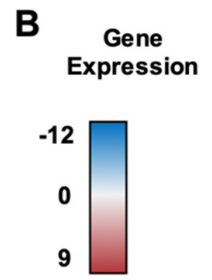

Log2(Fold-Change)

1097

C

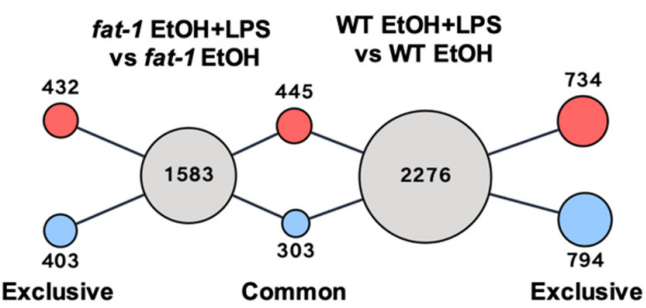

F

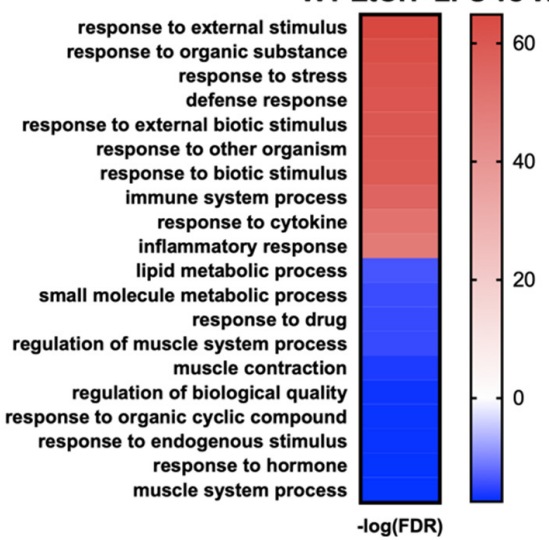

WT EtOH+LPS vs WT EtOH

1179

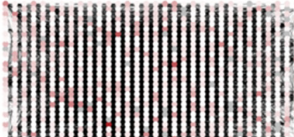

fat-1 EtOH+LPS vs fat-1 EtOH

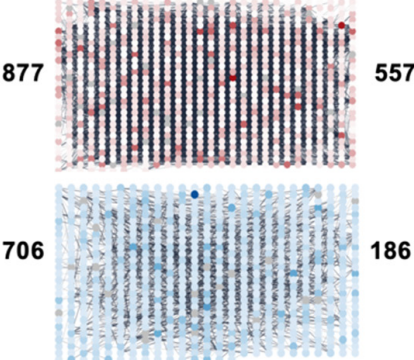

D WT EtOH+LPS vs WT EtOH

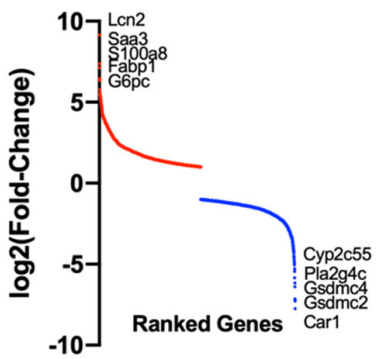

fat-1 EtOH +LPS vs WT EtOH+LPS

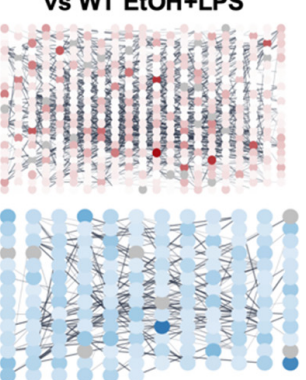

E

G
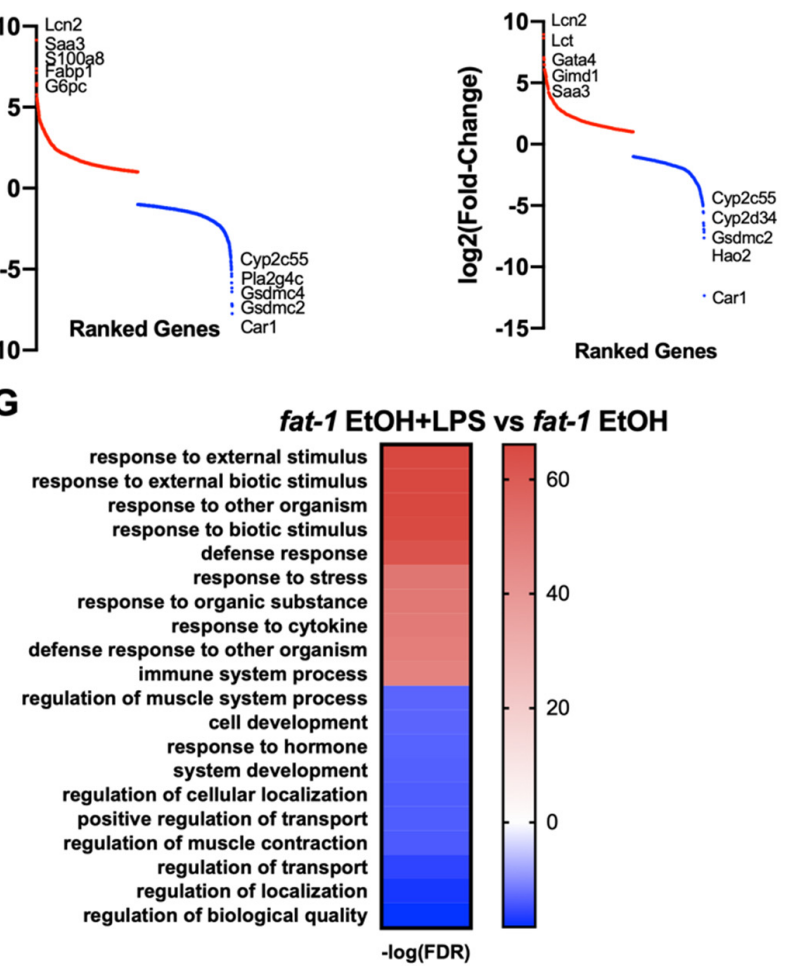

Figure 1. Chronic EtOH consumption followed by LPS challenge leads to global ileal gene changes in both WT and fat-1 mice. (A) WT and fat-1 mice were either pair-fed (WT $n=4$, fat-1 $n=4)$, EtOH-fed (WT $n=3$, fat- $1 n=5)$, or EtOH-fed + a one-time injection of LPS $24 \mathrm{~h}$ before sacrifice (WT-EtOH + LPS $n=4$, fat- $1 \mathrm{EtOH}+\mathrm{LPS} n=4)$. (B) Gross RNA-seq data from WT EtOH + LPS vs. WT EtOH mice, fat-1 EtOH + LPS vs. fat-1 EtOH mice, and fat-1 EtOH + LPS vs. WT EtOH + LPS mice. Nodes in the red gradient were increased for the given comparison and nodes in the blue gradient were decreased for the given comparison, with the total number of genes listed to the side. (C) The number of gene expression changes in response to EtOH + LPS, either exclusive or common to genotype. (D) Plot of $\log _{2}$ (Fold-change ranked genes) for the WT EtOH + LPS vs. WT EtOH comparison (red increased, blue decreased). (E) Plot of $\log _{2}$ (Fold-change ranked genes) for the fat $-1 \mathrm{EtOH}+\mathrm{LPS}$ vs. fat-1 EtOH comparison (red increased, blue decreased). (F) Heatmap of GO processes for the WT $\mathrm{EtOH}+\mathrm{LPS}$ vs. WT EtOH comparison. (G) Heatmap of GO processes for the fat-1 EtOH + LPS vs. fat-1 EtOH comparison. 


\subsection{Similarity in Transcriptional Responses of Ileum Tissue to EtOH+LPS in WT and fat-1 Mice}

Analysis of gene expression changes between WT and fat-1 mice revealed a number of similar changes in both. The most upregulated ileum genes in response to EtOH + LPS vs. EtOH in both genotypes included Lcn2, Gata4, Gimd1, Saa3, and Plb1 (496.8-, 134-, 123.9-, 122.2-, and 117.5-fold, respectively), while the genes with the greatest decreases included Car1, Cyp2c55, Hao2, Cyp2d34, and Gsdmc2 (-5168.5-, -197.9-, -143.9-, -127.1-, and -122.3fold, respectively) (Table S3). Cluster analysis identified gene groups that were similarly affected in EtOH + LPS vs. EtOH-treated WT and fat-1 mice. The expression of genes involved in cytokine signaling (Tnf, Il1b, Csf1), glucose metabolism (Aldob, Fbp1, Fbp2), glutathione metabolism (Gpx3, Gsta1, Ggt5), and nicotinamide adenine dinucleotide (NAD) metabolism (Nampt, Shmt2, Enpp1) were similarly enriched between genotypes (Figure 2A). Gene clusters with diminished expression included those for fatty acid metabolism (Fasn, Acaca, Scd1), smooth muscle contraction (Actg2, Myh11, Tpm2), xenobiotic metabolism (Cyp2e1, Ugt2b5, Cyp3a44), cell cycle (Mki67, Foxo3, Parp1), and $\mathrm{H}_{2} \mathrm{~S}$ metabolism (Flnc, Cbs, Mpst) (Figure 2B).

\section{Common Responses between fat-1 and WT EtOH-fed Mice to LPS Challenge}
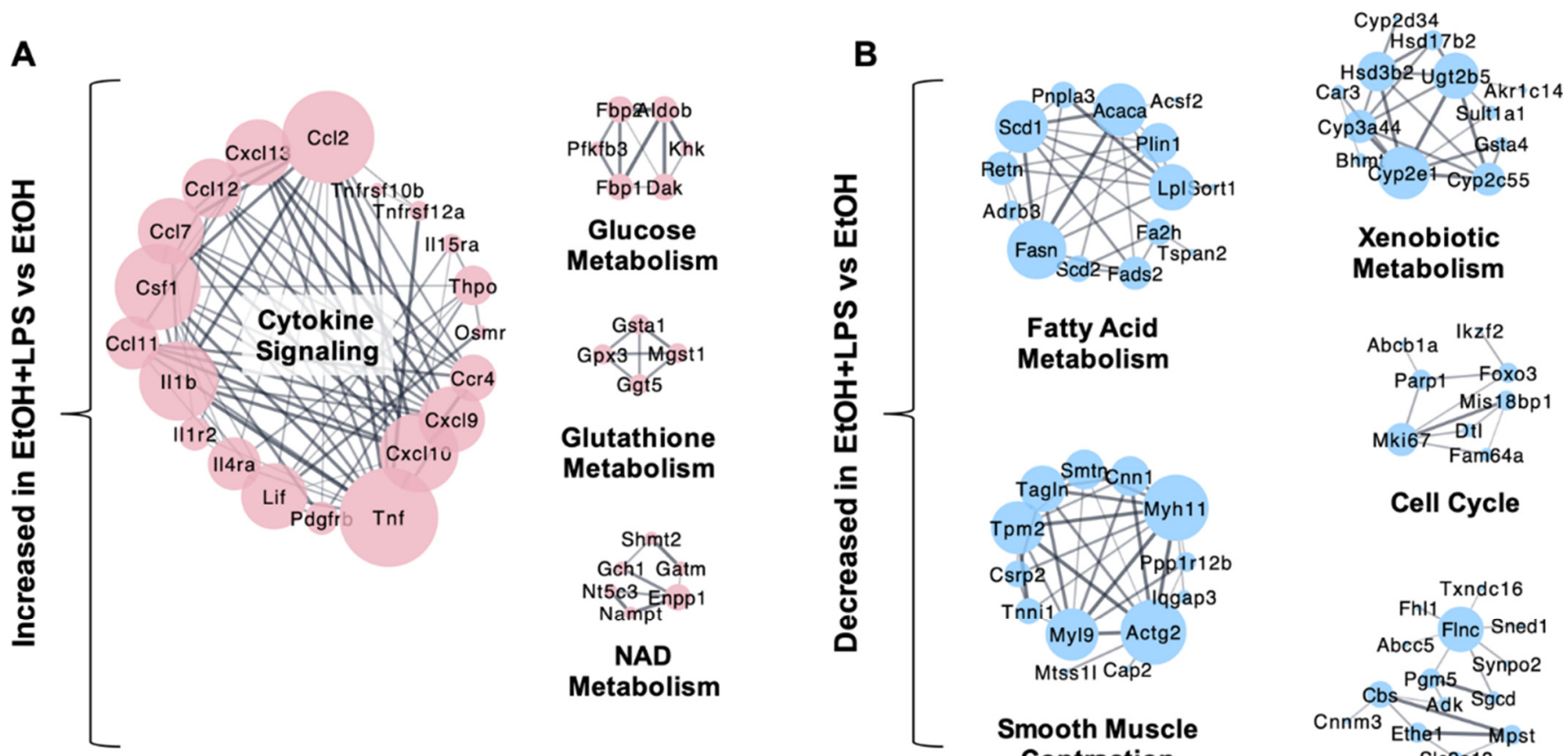

$$
\begin{aligned}
& \begin{array}{c}
\text { Xenobiotic } \\
\text { Metabolism }
\end{array} \\
& \text { Abcb1a Ikzf2 } \\
& \text { Parp1 Foxo3 } \\
& \text { Mis18bp1 } \\
& \text { Cell Cycle }
\end{aligned}
$$

Fatty Acid Metabolism
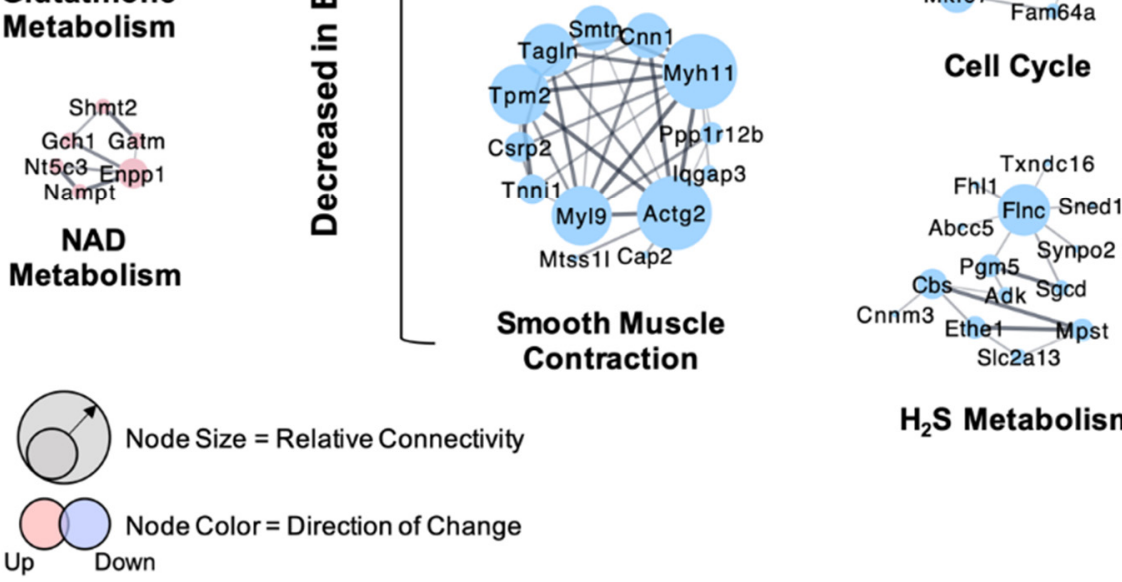

$\mathrm{H}_{2} \mathrm{~S}$ Metabolism

Figure 2. Similarity in transcriptional responses of ileum tissue to EtOH + LPS in WT and fat-1 mice. (A) Cluster analysis of ileum genes increased by EtOH + LPS vs. EtOH in both WT and fat-1 mice. Node size indicates relative connectivity. (B) Cluster analysis of ileum genes decreased by EtOH + LPS vs. EtOH in both WT and fat-1 mice.

\subsection{Increased Transcriptional Responses to EtOH + LPS Challenge Specific to WT or fat-1 Mice}

There were many transcriptional changes that were unique to each genotype. The most highly upregulated ileum genes exclusive to WT EtOH + LPS vs. WT EtOH-treated mice included Fabp1, S100a8, Igkv3-1, Ptx3, and Fga (166.6-, 80.7-, 47.5-, 44.6-, and 43.7fold, respectively) (Table S4). Ileum genes significantly upregulated exclusively in fat-1 $\mathrm{EtOH}+\mathrm{LPS}$ vs. fat-1 EtOH mice included Lct, Ugt2a3, Cyp2b10, Enpp3, and Fpr1(402-, 
91.6-, 64.6-, 53-, and 33.8-fold, respectively) (Table S5). Next, cluster analysis of genotypeexclusive gene sets in WT and fat-1 mice exposed to EtOH + LPS vs. EtOH was performed. Gene clusters upregulated in EtOH + LPS vs. EtOH-treated WT mice included those for immune response (Lilrb4, Cd14, Tyrobp), chemokine signaling (Cxcl1, Anxa1, Ccl9), peroxisome proliferator-activated receptor (PPAR) signaling (Ppard, Hmgcs2, Acaa1b), extracellular matrix (ECM) receptor interaction (Timp1, Thbs1, Adamts4), cytokine signaling (Il1a, Il1r1, Myd88), and ribosome biogenesis (Wdr12, Tdrd12, Utp23) (Figure 3A). Gene sets that were exclusively upregulated in EtOH + LPS vs. EtOH-treated fat-1 mice included those for lipid metabolism (Nr1h3, Apoc3, Srebf1), antigen presentation (Fpr1, H2-Q1, H2-Q2), amino acid (AA) metabolism (Cyp2b10, Cyp4a29, Gstm6), toll-like receptor (TLR) signaling (Ccl3, Ccl4, Nos2), innate immune response (Uba7, Adar, Atf3), and protein transport (Sec16b, Sar1b, Sec22b) (Figure 3B).

\section{Exclusive Responses (Up-Regulation) in fat-1 and WT EtOH-Fed Mice to LPS challenge}

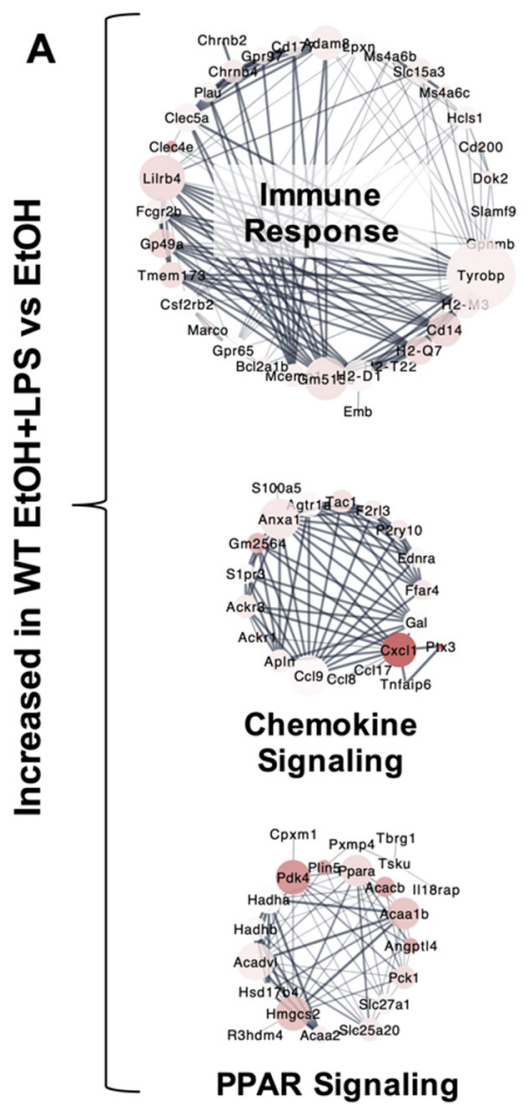

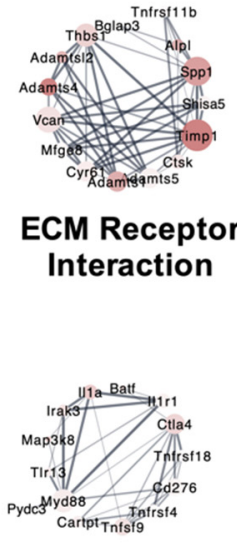

Cytokine

Signaling

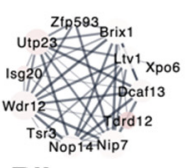

Ribosome

Biogenesis

$$
\text { Node Size = Relative Connectivity }
$$

Figure 3. Exclusive transcriptional responses increased due to EtOH + LPS in WT and fat-1 mice. (A) Cluster analysis of the expression of genes increased exclusively in WT mice in response to EtOH + LPS vs. EtOH. (B) Cluster analysis of ileum genes increased exclusively in fat-1 mice in response to EtOH + LPS vs. EtOH. Node size indicates relative connectivity. Node color indicates relative $\log _{2}$ (Fold-Change) of genes. 


\subsection{Decreased Transcriptional Responses to EtOH+LPS Challenge Specific to WT or fat-1 Mice}

There were also genes whose expression was decreased by EtOH + LPS vs. EtOH exclusively in WT and fat-1 mice. The most highly downregulated genes exclusive to WT EtOH + LPS vs. WT EtOH-treated mice included Mme, Igkv2-109, Igkv4-53, Ighv14-4, and Rn7sk (-33.1-, -31.2-, -24.5-, -19.8-, and -17.8-fold, respectively) (Table S4). Genes that were highly downregulated exclusively in fat-1 EtOH + LPS vs. fat-1 EtOH mice included Nov, Grin3a, Ighg2c, Npy4r, and Gm11346 (-86.8-, -45-, -32.8-, -29.2-, and -28.9-fold, respectively) (Table S5). Ileum gene clusters with decreased expression in WT mice in response to EtOH + LPS vs. EtOH included those for cell cycle (Top2a, Aurkb, Pola1), xenobiotic metabolism (Cyp3a11, Cyp2c68, Cyp3a44), Wnt signaling (Axin2, Fzd2, Nkd2), cyclic adenosine monophosphate (cAMP) signaling (Creb1, Pde5a, Fosb), and muscle contraction (Myl7, $M y l k, V c l$ ) (Figure 4A). Gene clusters with decreased expression exclusively in fat-1 mice in response to EtOH + LPS vs. EtOH included those for T cell signaling (Thy1, Ccr7, Cd8b), small molecule metabolism (Acly, Acaa1b, Pygb), mitogen-activated protein kinase (MAPK) signaling (Mapk10, Igf1r, Map2), phospholipase D signaling (Grp, Plcb1, Dhkh), protein glycosylation (B3gnt6, St3gal3, St6gal1), and ubiquitin proteolysis (Nedd4, Ubr2, Wwp1) (Figure 4B).

\section{Exclusive Responses (Down-Regulation) in fat-1 and WT EtOH-Fed Mice to LPS challenge}

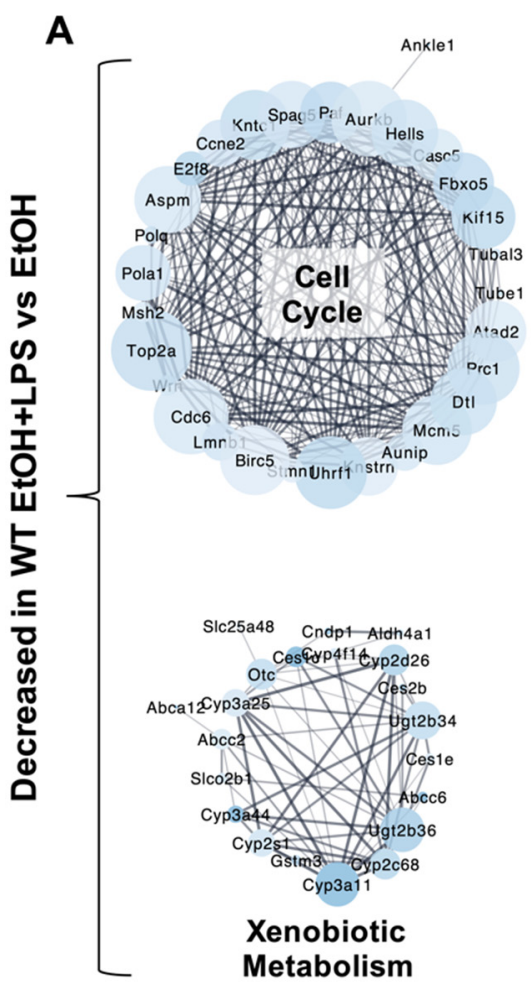

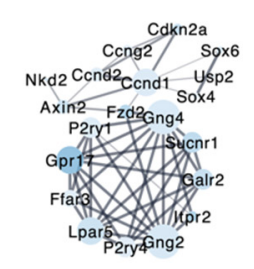

Wnt Signaling
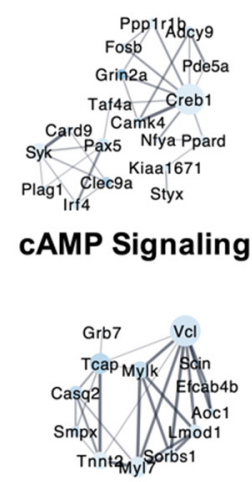

Muscle Contraction
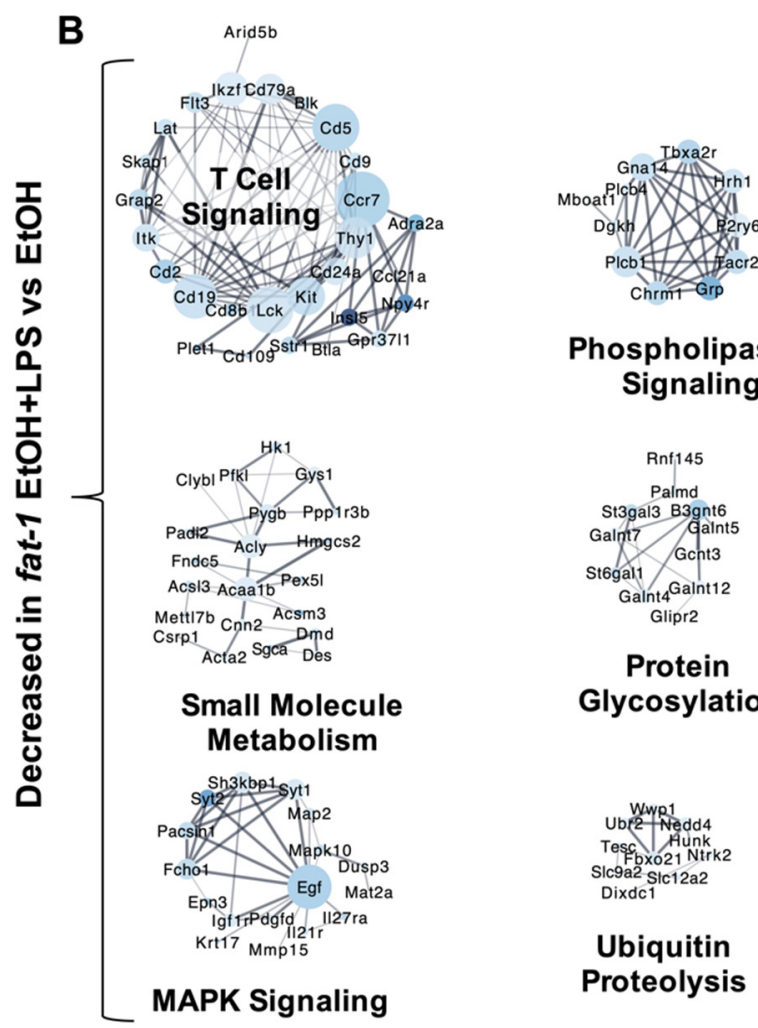

Phospholipase D Signaling

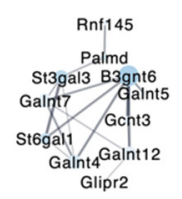

Protein Glycosylation

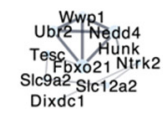

Ubiquitin

Proteolysis

\footnotetext{
Node Size = Relative Connectivity

$\bigcup_{-1}$ Node Color $=\log _{2}$ Fold Change
}

Figure 4. Exclusive transcriptional responses decreased due to EtOH + LPS in WT and fat-1 mice. (A) Cluster analysis of ileum genes decreased exclusively in WT mice in response to EtOH + LPS vs. EtOH. (B) Cluster analysis of ileum genes decreased exclusively in fat-1 mice in response to EtOH + LPS vs. EtOH. Node size indicates relative connectivity. Node color indicates relative $\log _{2}$ (Fold-Change) of genes. 


\subsection{Differential Transcriptional Responses between fat-1 EtOH+LPS and WT EtOH + LPS-Exposed Mice}

Cluster analysis of ileum genes differentially expressed between fat-1 EtOH + LPS vs. WT EtOH + LPS mice revealed several significant groups (Figure 5A). The cytokine signaling gene cluster was overrepresented for this comparison, which included the following genes: Il15, Il33, Serpine1 (increased), Cxcl13, Il6, and Itga5 (decreased). Ileum gene clusters enriched in fat-1 EtOH + LPS vs. WT EtOH + LPS mice also included genes for xenobiotic metabolism (Cyp2b10, Cyp3a11, Ugt2a3), phagosome (Mme, H2-Q10, H2-Q2), innate immune response (Oas2, Isg15, Cmpk2), and lipid metabolism (Agpat9, Dgat1, Soat2). The neuroactive ligand activation gene cluster had decreased expression of many genes in fat-1 EtOH + LPS vs. WT EtOH + LPS-treated mice, including Grp, Cck, and Gal. The most upregulated genes in fat-1 EtOH + LPS vs. WT EtOH + LPS-treated mice included Lct, Slc28a1, Cyp2b10, Mme, and Enpp3 (202.9-, 100.9-, 96.7-, 46.4-, and 45.9-fold, respectively) with the most downregulated genes being Ighg1, Hal, Fa2h, Igkv4-69, and Zfp865 (-18.6-, $-14.6-,-8-,-7.1-$, and -6.7-fold, respectively) (Table S6) (Figure 5B). GO processes enriched in fat-1 EtOH + LPS vs. WT EtOH + LPS mice included response to stress, response to hypoxia, and signaling receptor activity, while the diminished processes included anion transport, organophosphate metabolism, and carboxylic acid metabolism (Figure 5C).

\subsection{Targeted Analysis of Selected Transcriptional Responses Involved in EtOH + LPS-Mediated} Alterations of Intestinal Immunity and Intestinal Tissue Integrity

Specific gene sets were investigated to further delineate the effects of chronic $\mathrm{EtOH}$ administration in combination with acute inflammation, as well as the role of n-3 PUFA enrichment, on processes related to intestinal immunity and fibrotic intestinal tissue alterations, which are key drivers of intestinal homeostasis and pathology, respectively [1]. To this end, Butyrophilin-like (Btnl) genes, gene markers of intestinal $\gamma \delta \mathrm{T}$ cells, macrophages, $\operatorname{IgA}+\mathrm{B}$ cells, and drivers of intestinal tissue fibrosis were evaluated in fat- 1 and WT mice challenged with EtOH + LPS. We also evaluated effects of chronic EtOH exposure alone on specific mediators and pathways involved in intestinal immunity and fibrotic processes by comparing the pair-fed (PF) and EtOH-fed WT and fat-1 mice; these data are presented in Supplemental Materials Figures S2-S4.

2.6.1. Ileum Gene Expression of Butyrophilin-Like (Btnl) Genes and Markers of $\gamma \delta$ T Cells and Pro-Restorative Macrophages Were Enhanced in fat-1 vs. WT

EtOH + LPS-Exposed Mice

Butyrophilin-like (Btnl) genes have recently been demonstrated to be expressed by intestinal epithelial cells (IECs) where they act as tethers to enhance the $\gamma \delta$ T cell population and heighten defense response and tissue homeostasis [17,18]. The expression of Btnl1/2 and Tcrg-C1 (a $\gamma \delta \mathrm{T}$ cell marker) was decreased by EtOH in both WT and fat-1 mice (Figure S2A,B). Interestingly, all Btnl and $\gamma \delta \mathrm{T}$ cell signature genes (other than Btnl2) were decreased in EtOH + LPS vs. EtOH-treated WT mice. In contrast, the same genes (other than Btnl9) were increased by EtOH + LPS vs. EtOH in fat-1 mice (Figure 6A). Notably, the Btnl genes (Btnl1/2/4/6/9), Tcr-G1, and Tcrg-V7 were all elevated in fat-1 EtOH + LPS mice as compared to WT EtOH + LPS mice (Figure $6 \mathrm{~B}$ ). $\mathrm{T}_{h} 1, \mathrm{~T}_{\text {reg }}$, and $\mathrm{T}_{\mathrm{h}} 17$ cell gene markers were evaluated, but there were no statistical differences noted for WT EtOH + LPS vs. WT $\mathrm{EtOH}$, fat-1 EtOH+LPS vs. fat-1 EtOH (Figure 6C) or fat-1 EtOH + LPS vs. WT EtOH + LPS mice (Figure 6D).

Pro-restorative macrophages contribute to favorable intestinal tissue homeostasis and host immune responses [19]. Pro-restorative macrophage gene signatures were evaluated in the ileum of EtOH and PF WT and fat-1 mice. The expression of $C x 3 c l 1$ and Ltb4r1 was decreased by EtOH in both genotypes but Slpi was only decreased in WT mice (Figure S2C). The expression of pro-restorative macrophage gene signatures was elevated in the EtOH + LPS vs. EtOH group in both WT and fat-1 mice (except for Tek and Mertk) (Figure 6E). The expression of these pro-restorative macrophage genes was also increased in fat-1 EtOH + LPS vs. WT EtOH + LPS mice with Slpi, Mertk, and Ltb4r1 being significant 
(Figure 6F). Taken together, these data demonstrate that enhanced n-3 PUFAs increased ileum Btnls, $\gamma \delta \mathrm{T}$ cells, and pro-restorative MertK+ macrophages in EtOH + LPS treated mice (Figure 6G).

\section{fat-1 EtOH+LPS vs WT EtOH+LPS}

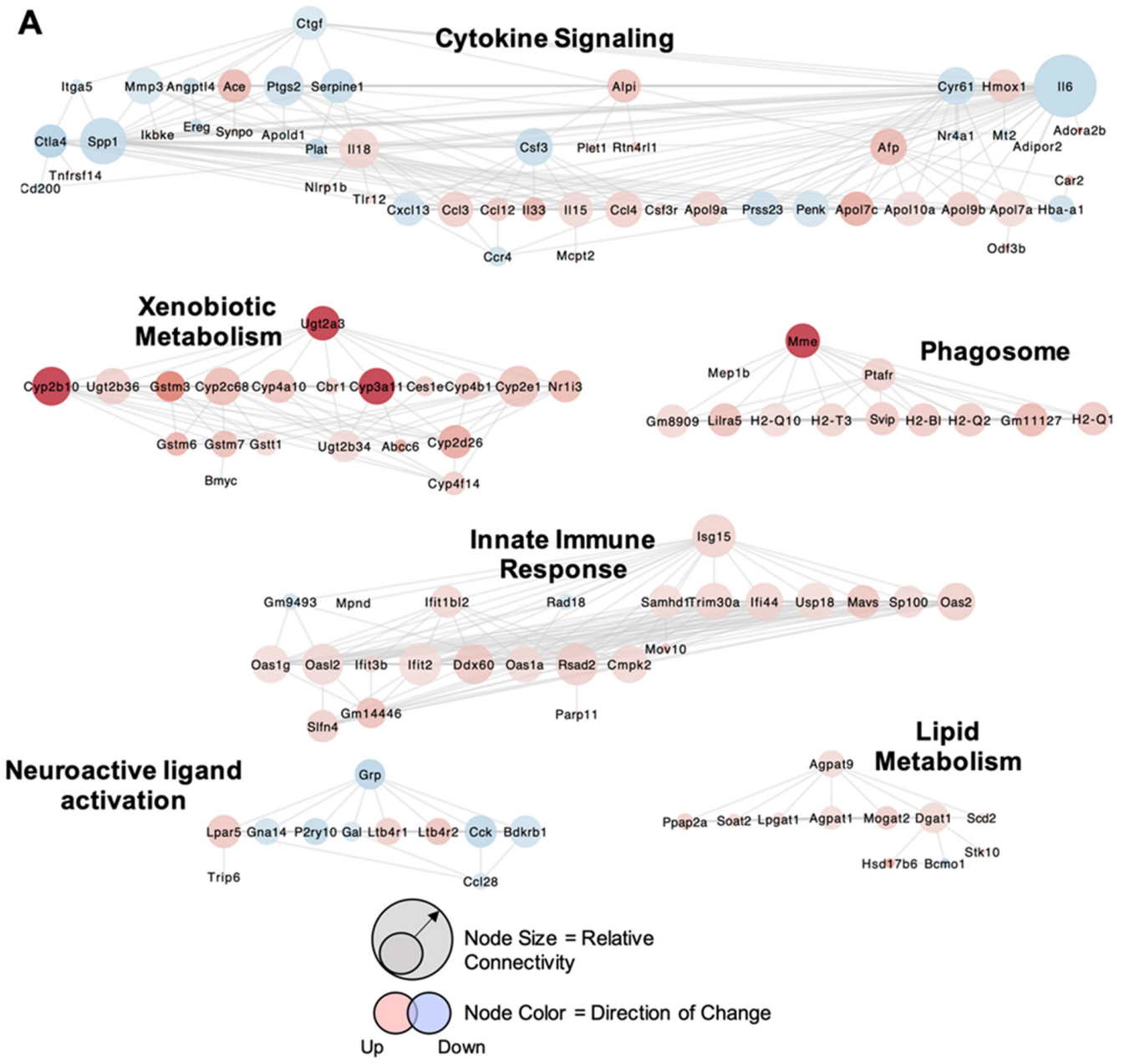

B
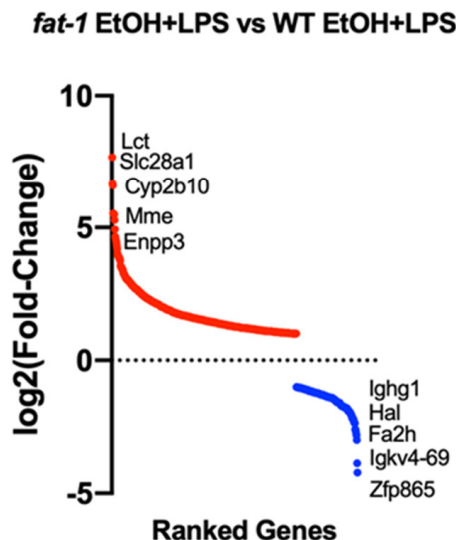

C

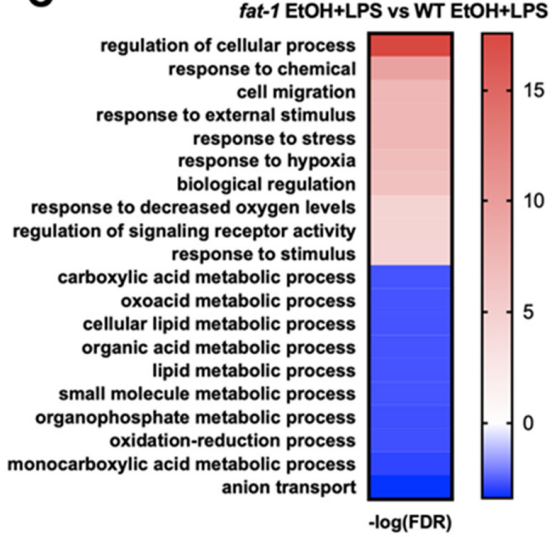

Figure 5. Differential transcriptional responses in fat-1 EtOH + LPS vs. WT EtOH + LPS-treated mice. (A) Cluster analysis of ileum genes differentially expressed between fat-1 EtOH + LPS vs. WT EtOH + LPS-treated mice. Node size indicates relative connectivity. Node color indicates relative $\log _{2}$ (Fold-Change) of genes. (B) Plot of $\log _{2}$ (Fold-change ranked genes) for the fat-1 EtOH + LPS vs. WT EtOH + LPS comparison (red increased, blue decreased). (C) Heatmap of GO processes for the fat $-1 \mathrm{EtOH}+$ LPS vs. WT EtOH + LPS comparison. 
A

\begin{tabular}{|c|c|c|}
\hline Gene & WT EtOH+LPS vs WT EtOH & fat-1 EtOH+LPS vs fat-1 EtOH \\
\hline Btn/1 & -1.99 & 1.87 \\
\hline Btn/2 & 1.29 & 4.15 \\
\hline Btn/4 & -1.41 & 1.37 \\
\hline Btn/6 & -1.71 & 1.53 \\
\hline Btn/9 & -2.35 & -1.67 \\
\hline Trg-C1 & -1.45 & 1.97 \\
\hline Tcrg-V7 & -3.16 & 2.38 \\
\hline
\end{tabular}

C

\begin{tabular}{|c|c|c|}
\hline Gene & WT EtOH+LPS vs WT EtOH & fat-1 EtOH+LPS vs fat-1 EtoH \\
\hline \multicolumn{3}{|c|}{$\mathrm{T}_{\mathrm{H}} 1 \mathrm{Markers}$} \\
\hline Ifng & 1.08 & -1.48 \\
\hline Stat4 & 2.38 & -1.34 \\
\hline$T b \times 21$ & -1.54 & -1.26 \\
\hline \multicolumn{3}{|c|}{$T_{\text {reg }}$ Markers } \\
\hline$T g f b 1$ & 1.53 & 1.16 \\
\hline Foxp3 & 1.02 & -3.30 \\
\hline IIIra & -1.37 & -3.04 \\
\hline \multicolumn{3}{|c|}{$T_{H} 17$ Markers } \\
\hline II17f & -1.31 & -1.04 \\
\hline Stat3 & 1.26 & 1.46 \\
\hline Rorc & -1.20 & 1.30 \\
\hline
\end{tabular}

E

\begin{tabular}{|c|c|c|c|c|}
\hline \multirow{2}{*}{$\begin{array}{l}\text { Gene } \\
\text { F4/80 }\end{array}$} & \multicolumn{2}{|c|}{ WT EtOH+LPS vs WT EtOH } & \multicolumn{2}{|c|}{ fat-1 EtOH+LPS vs fat-1 EtOH } \\
\hline & 1.58 & $\star$ & 2.04 & * \\
\hline Cd64 & 5.76 & * & 5.10 & * \\
\hline Tek & -2.10 & * & -1.25 & \\
\hline SIpi & 3.39 & * & 5.45 & * \\
\hline Mertk & -1.85 & * & 1.54 & * \\
\hline Ltb4r & 3.21 & * & 14.45 & * \\
\hline$C \times 3 c / 1$ & 1.00 & & 1.43 & * \\
\hline $\mathrm{C} \times 3 \mathrm{cr}$ & 1.08 & & 1.10 & \\
\hline
\end{tabular}

B

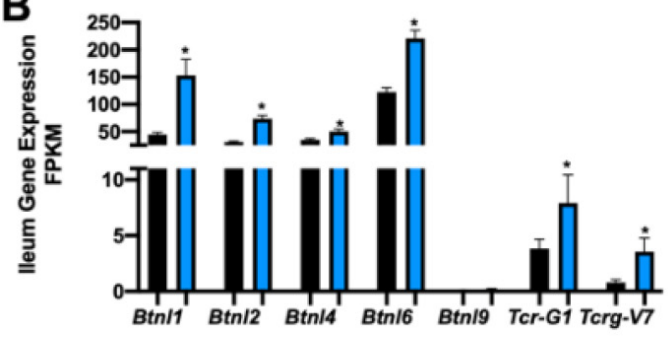

D
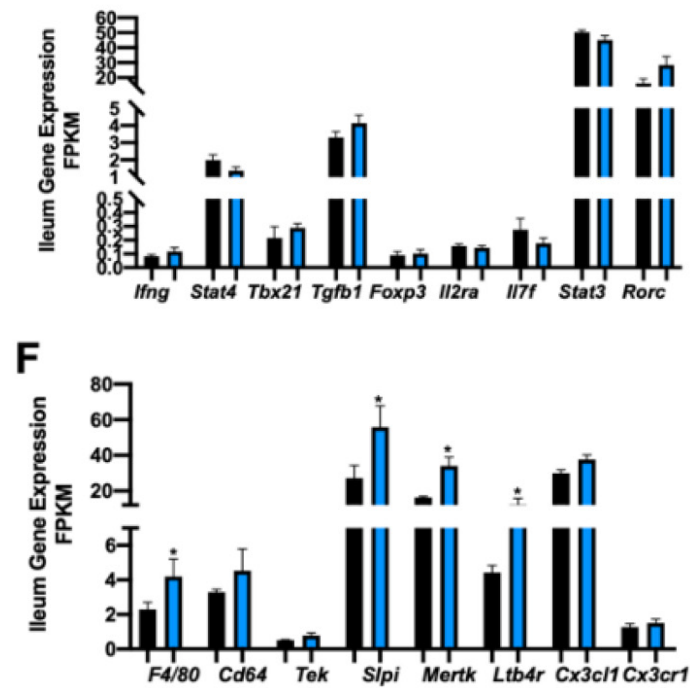

G

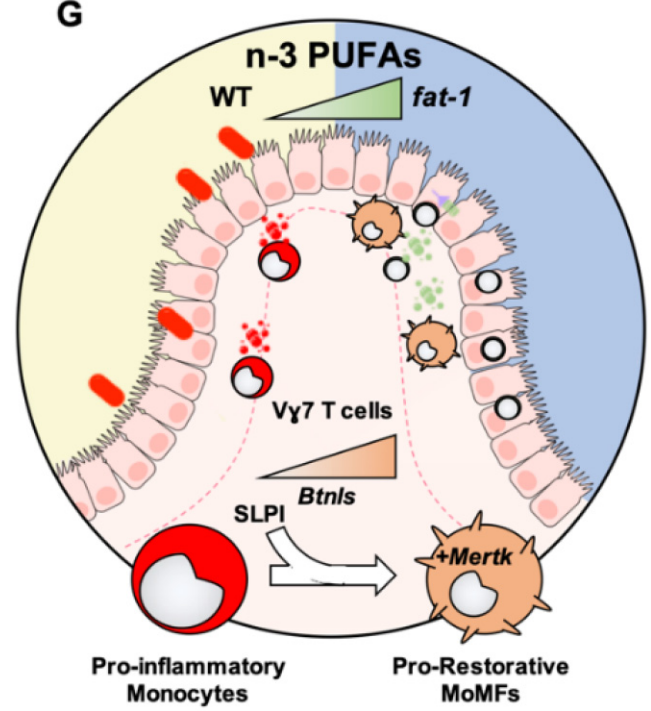

Figure 6. Increased n-3 PUFAs enhanced the ileum expression of Btnl-mediated $\mathrm{T}$ cell and pro-restorative macrophage gene signatures. (A) Heatmap fold change values for ileum Btnl and $\gamma \delta \mathrm{T}$ cell gene signatures for the WT EtOH + LPS vs. WT EtOH comparison and the fat-1 EtOH + LPS vs. fat- $1 \mathrm{EtOH}$ comparison. (B) Ileum Btnl and $\gamma \delta \mathrm{T}$ cell gene signature expression for WT EtOH + LPS and fat-1 EtOH + LPS-treated mice. (C) Heatmap fold-change values for $\mathrm{T}_{\mathrm{h}} 1, \mathrm{~T}_{\mathrm{reg}}$, and $\mathrm{T}_{\mathrm{h}} 17$ cell gene markers for the WT EtOH + LPS vs. WT EtOH comparison and the fat-1 EtOH + LPS vs. fat- $1 \mathrm{EtOH}$ comparison. (D) Gene expression of $\mathrm{T}_{\mathrm{h}} 1, \mathrm{~T}_{\text {reg, }}$, and $\mathrm{T}_{\mathrm{h}} 17$ cell gene markers in WT EtOH + LPS vs. fat-1 EtOH + LPS-treated mice. (E) Heatmap fold change values for ileum pro-restorative macrophage markers. (F) Pro-restorative macrophage gene expression for WT EtOH + LPS and fat-1 EtOH + LPS-treated mice. (G) Graphical representation of BTNLs, $\gamma \delta$ T cells, and pro-restorative macrophages being enhanced in the ileum by n-3 PUFA enrichment. Statistical significance $(p<0.05)$ is denoted by an * 


\subsubsection{Increased n-3 PUFAs Enhanced APRIL Gene Expression and IgA + B-Cell Markers}

Next, we evaluated APRIL signaling, an important pathway in intestinal B cellmediated anti-bacterial response [20]. Activation of intestinal B cells by APRIL (Tnfsf13) signaling leads to the production of IgA which functions to mitigate bacterial expansion in the gut [21]. APRIL signals through its receptors TACI (Tnfrsf13b) and BCMA (Tnfrs17), which, in turn, switches B cells to IgA producing B cells [21]. The expression of Tnfrsf17, Igha, and Jchain was decreased by EtOH feeding in both genotypes while Tnfsf 13 was only decreased in WT EtOH mice (Figure S3). The expression of Tnfrsf13b was increased in fat-1 EtOH vs. WT EtOH mice (Figure S3). Tnfsf13, Tnfrsf13b, Tnfrsf17, Igha, and Jchain gene expression was decreased by EtOH + LPS in both genotypes, except for Igha and Jchain, which were elevated in fat-1 EtOH + LPS vs. fat- $1 \mathrm{EtOH}$ mice (Figure 7A). When directly comparing the expression of these genes between fat-1 EtOH + LPS vs. WT EtOH + LPStreated mice, all were increased in fat-1 mice (with Tnfsf13, Igha, and Jchain being significant) (Figure 7B). Collectively, these data suggest that enrichment of n-3 PUFAs increased IgAproducing $\mathrm{B}$ cells and APRIL signaling markers in the intestine of EtOH + LPS treated fat-1 mice (Figure 7C).

A

\begin{tabular}{|c|c|c|}
\hline Gene & WT EtOH+LPS vs WT EtOH & fat-1 EtOH+LPS vs fat-1 EtOH \\
\hline Tnfsf13 & $-3.08 *$ & -1.26 \\
\hline Tnfrsf13b & -1.20 & -1.69 \\
\hline Tnfrsf17 & -14.93 & -1.29 \\
\hline Igha & $-1.46 *$ & 2.01 \\
\hline Jchain & $-2.23 *$ & 1.25 \\
\hline
\end{tabular}

B

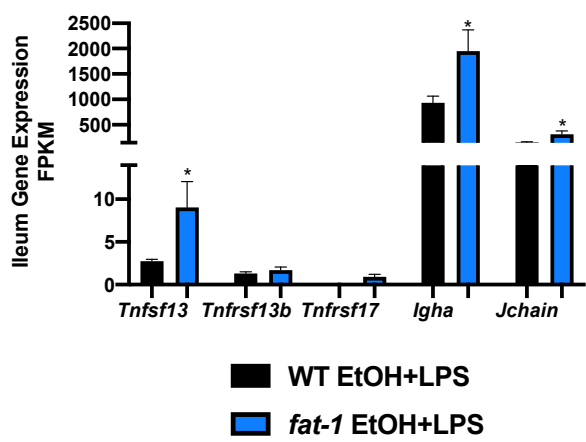

C

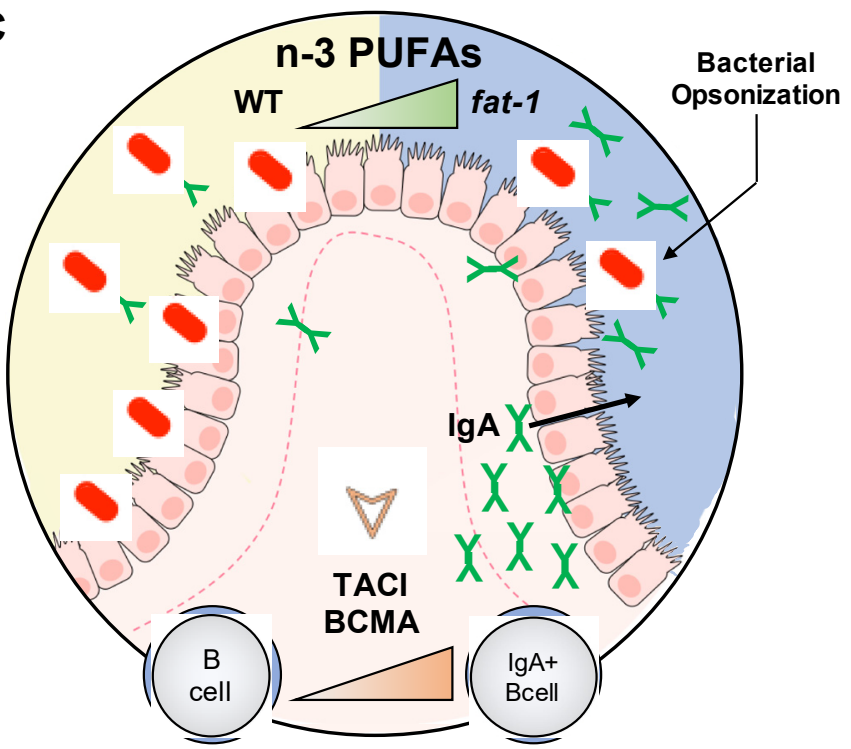

Figure 7. Increased n-3 PUFAs enhanced APRIL-signaling gene expression and IgA + B-Cell markers. (A) Heatmap fold-change values for APRIL signaling genes and IgA genes for the WT EtOH + LPS vs. WT EtOH comparison and the fat-1 EtOH + LPS vs. fat-1 EtOH comparison. (B) APRIL signaling and IgA gene expression for WT EtOH + LPS and fat-1 EtOH + LPS-treated mice. (C) Graphical representation of enhanced APRIL signaling and IgA + B cells in the ileum of mice with n-3 PUFA enrichment. Statistical significance $(p<0.05)$ is denoted by an *.

2.6.3. Increased n-3 PUFAs Attenuated the EtOH+LPS-Mediated Intestinal Pro-Fibrotic Gene Expression

Chronic intestinal injury and inflammation may result in intestinal fibrosis [22]. Thus, we evaluated the expression of the pro-fibrotic receptors Pdgfra, Tgfbr2, and Igfr 1 , which was increased by EtOH independent of genotype (Figure S4A), along with Acta2, an established marker of fibrosis (Figure S4B). Next, when comparing EtOH + LPS vs. EtOH in WT and fat-1 mice, the expression of Pdgfra, Tgfbr2, Tgfbr3, Mmp3, and Lox was increased in both genotypes. The expression of Igf1r and Acta2 was decreased by EtOH + LPS in both genotypes. Fgfr 1 and Ctg $f$ were increased in WT mice but decreased in fat-1 mice in response to EtOH + LPS vs. EtOH (Figure 8A). When directly comparing fat-1 EtOH + LPS vs. WT EtOH + LPS mice, the expression of the pro-fibrotic receptors (Fgfr1, Pdgfra, Tgfbr2, $T g f b r 3$, Igf1r) was decreased in fat-1 mice (Figure 8B). Pro-fibrotic gene signatures (Mmp3, 
Ctgf, Lox, Acta2) were downregulated in fat-1 EtOH + LPS vs. WT EtOH + LPS-treated mice (Figure 8C). To histologically assess ileum fibrosis, Sirius red staining was performed, which revealed a lower extent of fibrotic scarring (although not significantly so) in fat-1 $\mathrm{EtOH}+\mathrm{LPS}$ relative to WT EtOH + LPS-treated mice (Figure 8D,E). In WT EtOH fed mice there was a significant increase in Sirius red staining relative to WT PF that was not observed in fat-1 EtOH vs. fat-1 PF mice (Figure S4C,D). Collectively, these data demonstrate that endogenous elevation of n-3 PUFAs diminished the expression of EtOH + LPS-induced intestinal fibrosis markers (Figure 8F).

A

\begin{tabular}{|c|c|c|c|}
\hline Gene & WT EtOH+LPS vs & $\mathrm{EtOH}$ & fat-1 EtOH+LPS vs fat-1 EtOH \\
\hline Fgfr & 1.50 & * & -1.12 \\
\hline Pdgfra & 1.74 & * & 1.28 \\
\hline Tgfbr2 & 2.27 & * & 1.06 \\
\hline Tgfbr3 & 2.22 & * & 1.12 \\
\hline Igf1r & -1.32 & & -2.37 \\
\hline Mmp3 & 34.02 & * & 12.15 \\
\hline Ctgf & 2.61 & * & -1.54 \\
\hline Lox & 33.22 & * & 8.72 \\
\hline Acta2 & -1.22 & & -2.85 \\
\hline
\end{tabular}

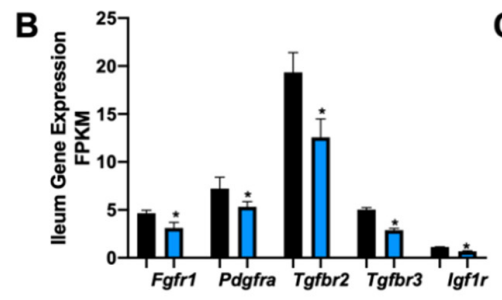

C

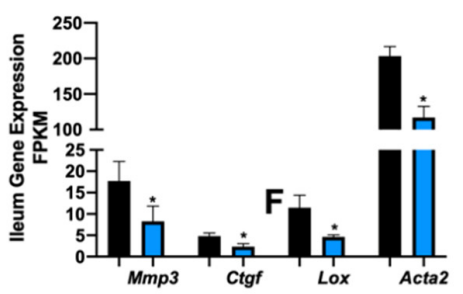

D

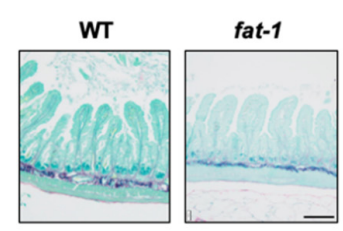

EtOH+LPS

E

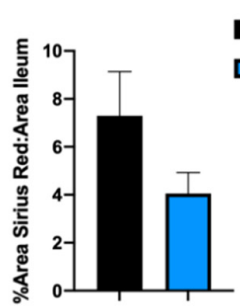

F

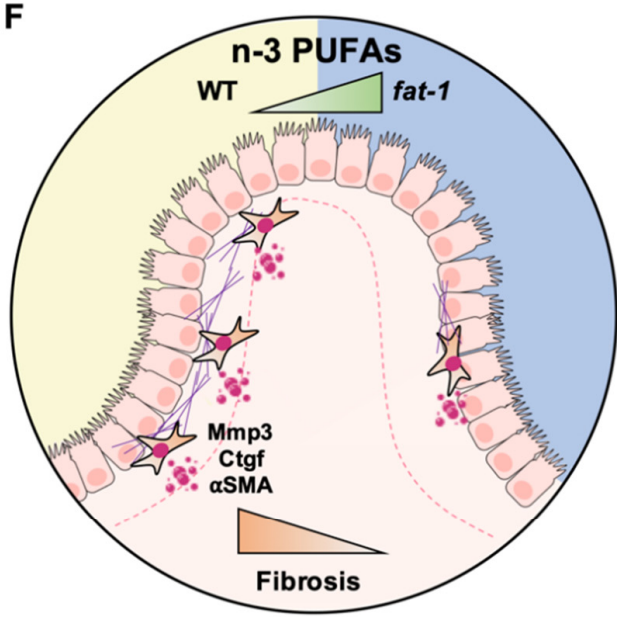

WT EtOH+LPS

$\square$ fat-1 EtOH+LPS

Figure 8. Increased n-3 PUFAs attenuated the EtOH + LPS mediated intestinal fibrosis. (A) Heatmap fold-change values for pro-fibrotic receptors and markers for the WT EtOH + LPS vs. WT EtOH comparison and the fat-1 EtOH + LPS vs. fat-1 EtOH comparison. (B) Gene expression of pro-fibrotic receptors in WT EtOH + LPS and fat-1 EtOH + LPS-treated mice. (C) Gene expression of pro-fibrotic markers in WT EtOH + LPS and fat-1 EtOH + LPS-treated mice. (D) Representative Images of Sirius red-stained ileal sections at $200 \times$ from WT EtOH + LPS and fat-1 EtOH + LPS-treated (scale bar is $40 \mu \mathrm{m}$ ).

(E) Quantification of Sirius red staining area relative to total ileum area for WT EtOH + LPS vs. fat-1 EtOH + LPS mice.

(F) Graphical representation of diminished intestinal fibrosis associated with enhanced n-3 PUFAs. Statistical significance $(p<0.05)$ is denoted by an *.

\section{Discussion}

The detrimental effects of chronic alcohol exposure on intestinal tissue integrity and function are well known [11,23]. Specifically, excessive alcohol consumption is often associated with endotoxemia [24] as a result of increased gut permeability and leakage of gut-derived pathogens, including LPS, into circulation, affecting multiple tissues and organs including the gut. However, the underlying mechanisms of EtOH and EtOH + LPSmediated gut alterations are not well understood. In this study, we aimed to identify global transcriptional changes in the intestine following chronic EtOH exposure and LPS-induced systemic inflammation. In order to examine the effects of n3 PUFAs in modulating the transcriptional responses, we utilized fat-1 transgenic mice with endogenously elevated n-3 PUFA levels in all tissues. We observed that in response to EtOH + LPS vs. EtOH alone, WT mice experienced the greatest differential change in gene expression (2276 DEGs for WT vs. 
1583 for fat-1). WT and fat-1 mice had some similar transcriptional responses to EtOH + LPS vs. EtOH, including elevated Lcn2 (the most induced gene in both genotypes), which is pro-inflammatory and recruits immune cells to sites of inflammation [25]. The expression of other inflammatory mediators was similarly increased in both genotypes, including Tnf, Illb, and Csf1 in the EtOH + LPS vs. EtOH group. Csf1 is required to maintain the resident intestinal macrophage population [26], and these data suggest that in an EtOHacute systemic inflammation model there is an increase in resident intestinal macrophages, independent of genotype. Further, the expression of smooth muscle contraction genes, including Actg2, was decreased in EtOH + LPS vs. EtOH mice in both genotypes as well. Loss of function mutations of Actg2 lead to reduced intestinal peristalsis and correlate with disease severity in chronic intestinal pseudo-obstruction syndromes [27]. These data indicate that $\mathrm{EtOH}+\mathrm{LPS}$ can reduce the expression of intestinal motility markers independent of n-3 PUFA enrichment; therefore, EtOH and LPS may promote a loss of intestinal peristalsis. LPS has been shown to disturb intestinal motility in a JNK dependent manner through upstream TNF $\alpha$ signaling [28]. The TNF $\alpha$-JNK pathway may be less active in fat-1 mice in response to EtOH + LPS, restoring intestinal motility, but future investigation is required to validate this concept.

WT and fat-1 mice also had differential responses to EtOH + LPS vs. EtOH alone, including increased expression of chemokine and cytokine signaling genes in WT mice. Inflammation of the gut is common with chronic alcohol consumption, inflammatory bowel disease (IBD), and colitis. For example, Cxcl1, a chemokine that recruits neutrophils and is highly expressed in IBD [29], was highly expressed in WT but not fat-1 mice in response to EtOH + LPS. Similarly, both IBD-associated pro-inflammatory mediators, Il1a and its receptor Il1r [30], were increased exclusively in WT EtOH + LPS-treated mice. These data indicate that $\mathrm{n}-3$ PUFAs may function to dampen the intestinal immune response to $\mathrm{EtOH}$ and LPS. In contrast, the expression of lipid metabolism transcription factors such as Nr1h3 and Srebf1 were increased in only fat-1 mice in response to EtOH+LPS vs. EtOH. Deficient intestinal lipid metabolism has previously been described in IBD [31]. Recently, the loss of Nr1h3 was shown to exacerbate experimental colitis, and NR1H3 expression was found to be reduced in IBD patients [32]. Therefore, endogenous enrichment of n-3 PUFAs may transcriptionally regulate lipid metabolism in the intestine via increased expression of Nr1h3 which in turn elevates the expression of its target gene Srebf1.

There were also genesets decreased exclusively in the EtOH + LPS vs. EtOH groups in each genotype. The expression of genes involved in Wnt signaling were exclusively decreased in WT mice in response to EtOH + LPS, including Axin2, a transcriptional readout of Wnt signaling. Wnt signaling in intestinal stem cells is required for damage-induced regeneration of the intestinal epithelium (as in this EtOH-acute systemic inflammation model), but not for normal intestinal homeostasis [33]. Our data would suggest that n-3 PUFAs enhance the proliferative capacity of the intestinal epithelium during an EtOHacute systemic inflammation model. Other ileum genes were exclusively decreased in fat-1 $\mathrm{EtOH}+\mathrm{LPS}$ vs. EtOH-treated mice. The expression of cytotoxic $\mathrm{T}$ cell markers, including Thy 1 and $C d 8 b$ was decreased exclusively in EtOH + LPS vs. EtOH fat-1 mice. Cytotoxic T cells are elevated in IBD [34] and may exacerbate intestinal inflammation. Enrichment of n-3 PUFAs may function to reduce cytotoxic $\mathrm{T}$ cells stimulated by EtOH and LPS.

When directly comparing fat-1 EtOH + LPS vs. WT EtOH + LPS-treated mice, we found an increase in the expression of xenobiotic metabolism genes in fat-1 mice, including Cyp2b10, Cyp3a11, and Nr1i3. Cyp2b10 and Cyp3a11, both of which are target genes for Nr1i3, are decreased in expression in ulcerative colitis [35]. Enrichment of n-3 PUFAs may increase the expression and transcriptional activity of $N r 1 i 3$, providing a robust xenobiotic metabolism response during EtOH and LPS-induced injury and inflammation. The expression of intestinal Serpine1 was decreased in fat-1 EtOH + LPS vs. WT EtOH + LPS mice as well. SERPINE1 is elevated in IBD patients and links intestinal inflammation to coagulation, both of which are both factors involved in fibrosis [36]. Consumption of n-3 
PUFAs in humans has been demonstrated to reduce SERPINE1 in whole blood [37], but it may have similar effects on the intestine, as suggested by these data.

Previously we demonstrated that n-3 PUFA enrichment in fat-1 mice reduced proinflammatory cytokine levels in the ileums of EtOH + LPS-exposed mice [15]. Here we report that the expression of pro-inflammatory markers was reduced in fat-1 mice in contrast to pro-restorative immune cell markers, which were increased. Intraepithelial $\gamma \delta$ $\mathrm{T}$ cells play a pro-restorative role in intestinal immune responses and tissue homeostasis. The expression of $\gamma \delta \mathrm{T}$ cells markers (Tcrg-C1, Tcrg-V7) was increased with elevated n-3 PUFAs after EtOH + LPS treatment. The effect of fat- 1 appears to be specific to ileum $\gamma \delta \mathrm{T}$ cells markers as other $T$ cell population markers $\left(T_{h} 1, T_{h} 17, T_{\text {reg }}\right)$ were not altered. Btnl gene expression was also elevated by n-3 PUFA enrichment. BTNLs are expressed by IECs and enhance the recruitment of intraepithelial $\gamma \delta \mathrm{T}$ cells [17]. Intestinal mucosa $\gamma \delta$ $\mathrm{T}$ cells regulate immunotolerance to pathogenic bacteria and promote intestinal wound healing [38]. Metabolites of n-3 PUFAs have been demonstrated to impact $\mathrm{T}$ cell functions but not $\gamma \delta$ T cells specifically [39]. The increased expression of $\gamma \delta \mathrm{T}$ cell markers may be due to a n-3 PUFA effect on IEC Btnl gene expression. The transcriptional regulation of $B t n l$ genes is understudied, but n-3 PUFAs may transcriptionally regulate $B t n l$ gene expression through nuclear receptor activation [40]. IECs expressing more Btnls due to n-3 PUFA enrichment could explain the retention of $\gamma \delta \mathrm{T}$ cells. Enriched intestine $\gamma \delta \mathrm{T}$ cells may contribute to intestinal integrity and diminished inflammation. The expression of markers of monocyte-derived macrophages (MoMFs), which are pro-restorative immune cells, was increased in fat-1 mice. MoMFs contribute to intestinal homeostasis and resolution of inflammation. Recently, a dysfunction in monocyte differentiation to MoMFs has been demonstrated in IBD [19]. Infiltrating monocytes can receive external signaling cues and differentiate to MoMFs at sites of inflammation; one such cue is SLPI. SLPI increases the number of MERTK+ pro-restorative macrophages [41]. The expression of both Slpi and Mertk was elevated in fat-1 EtOH+LPS vs. WT EtOH+LPS-treated mice. It has been demonstrated that both Mertk $^{-/}$[42] and Slpi-- [43] mice develop exacerbated intestinal inflammation. Our data suggest that n-3 PUFAs may enhance the pool of pro-restorative macrophages to help maintain tissue homeostasis and integrity.

Enrichment of n-3 PUFAs also affected other intestinal immune cell responses. Intestinal immune cells generate many antibodies, such as $\operatorname{IgA}$, to mitigate bacterial overgrowth and maintain immune tolerance [44]. Secretory IgA is produced by B cells [45], which then undergoes transcytosis to reach the gut mucosal layer where it functions through immune exclusion to keep bacteria within the mucosa. Along with B cell-mediated prevention of bacterial translocation, the intestinal epithelial layer also maintains a physical barrier limiting gut permeability (e.g., ZO-1, and other tight junction proteins). Previously we found no prominent differences in intestinal tight junction genes or a reduction of plasma endotoxin in fat-1 EtOH fed mice relative to WT EtOH fed mice [15]. In the current model mice were fed an EtOH containing diet and LPS was administered systemically, which can on its own disrupt gut barrier function [13]. In this study there were no differences in ileum tight junction genes (Tjp1, Cldn, Ocln) between fat-1 EtOH + LPS vs. WT EtOH + LPS mice (data not shown). Here, we demonstrated that n-3 PUFA enrichment increased the expression of Igha and Jchain (the secretory IgA dimer linker) in the ileum. The expression of IgA + B cells is enhanced by APRIL (Tnfsf13) signaling [21]. APRIL is released by IECs following TLR activation (e.g., LPS activation of TLR4), leading to B cell proliferation and IgA secretion [21]. In this study, we found that the expression of APRIL (Tnfsf13) was increased by n-3 PUFA enrichment, which may contribute to the observed effect on Igha expression. Previously, it has been shown that the dietary n-3 PUFAs eicosapentaenoic acid and docosahexaenoic acid can enhance cecal IgA levels [46], consistent with the findings of this study. It has also been demonstrated that chronic EtOH consumption reduces fecal levels of secretory IgA in mice [47]. In an alcohol-associated liver disease mouse model, Igha ${ }^{-/}$mice did not develop exacerbated liver injury, which was partly explained by a compensatory upregulation of IgM [48], although intestinal pathology was not evaluated 
in this study. These data suggest that n-3 PUFAs enhanced expression of APRIL signaling components (ligand and receptors), which leads to enhanced IgA + B cell levels. This in turn would combat bacterial overgrowth and reduce bacterial-induced inflammation in the intestine.

In our study, fibrotic scarring of the ileum was increased by EtOH + LPS to a greater extent in WT vs. fat- 1 mice. Further, the expression of ileum pro-fibrotic receptors Tgfbr2/3, Pdgfra, Fgfr1 and Igf1r was decreased in fat-1 EtOH + LPS vs. WT EtOH + LPS-treated mice along with Ctgf, Mmp3, Lox, and Acta2. LPS- and microbe-induced intestinal fibrosis has been demonstrated previously [14], and here we demonstrate that elevation of n-3 PUFAs can reduce this effect. Intestinal fibrosis occurs after repeated intestinal injury and inflammation leading to a buildup of scar tissue [22]. This in turn stiffens the intestinal tract reducing gut motility, while simultaneously exacerbating permeability and GI bleeds [22]. Myofibroblasts in the intestine proliferate during intestinal fibrosis due to pro-fibrotic growth factors such as CTGF [49]. Interestingly, Ctgf expression was reduced with elevated n-3 PUFAs which could contribute to the decreased expression of other myofibroblast markers (Acta2, Lox, and Mmp3). In hepatic stellate cells, n-3 PUFAs reduce Ctg $f$ expression induced by $\mathrm{EtOH}$ [50]; here we show that this effect may also be present in intestinal stellate cells. Taken together, these data demonstrate that n-3 PUFAs are anti-fibrotic in the intestine during EtOH + LPS-mediated intestinal inflammation.

\section{Materials and Methods}

\subsection{Experimental Study Design}

Animal studies were approved by and performed in accordance with the guidelines of the University of Louisville Institutional Animal Care and Use Committee (IACUC). The IACUC protocol numbered 15423 was approved on 12 January 2016 by the IACUC ethics committee. Mice were housed in a temperature-controlled room with a $12 \mathrm{~h}$ light-dark cycle in a pathogen-free animal facility accredited by the Association for Assessment and Accreditation of Laboratory Animal Care. The fat-1 transgenic mouse line [5] was used to investigate the effect of n-3 PUFA enrichment on EtOH and LPS-induced intestinal pathology. These mice express the $C$. elegans fat- $1 \mathrm{n}-3$ fatty acid desaturase in all tissues and therefore have increased n-3 PUFAs in the absence of dietary modification [15]. 8-10-week-old fat-1 and WT male littermates were placed on a control (maltose dextrin) or EtOH-containing Lieber-DeCarli liquid diet (catalog numbers F1259SP and F1258SP, respectively. BioServ, Flemington, NJ, USA). Mice were fed for 6 weeks with an initial stepwise increase in EtOH concentration $(0 \%, 1 \%$, and $2 \%$ for two days each, $4 \%$ and $5 \%$ for one week each and then $6 \%$ for 3 weeks). EtOH-fed WT and fat-1 mice were subjected to a one-time i.p. injection of LPS $(5 \mathrm{mg} / \mathrm{kg}) 24 \mathrm{~h}$ before sacrifice to induce systemic inflammation (Figure 1A). RNASeq analysis of ileum tissue from WT PF $(n=4)$, fat-1 PF $(n=4)$, WT EtOH fed $(n=3)$, fat-1 EtOH fed $(n=5)$, WT EtOH + LPS $(n=4)$, and fat- $1 \mathrm{EtOH}+\mathrm{LPS}(n=4)$ experimental mice was conducted and analyzed in this study.

\subsection{Ileum Tissue Sample Acquisition and Histological Analysis}

Mice were anesthetized with ketamine/xylazine $(100 / 16 \mathrm{mg} / \mathrm{kg})$ and ileum tissue was collected and either snap-frozen in liquid nitrogen and stored at $-80^{\circ} \mathrm{C}$ for further RNA isolation, or immediately fixed in $10 \%$ neutral buffered formalin and embedded in paraffin for Picrosirius red staining as a measure for intestinal fibrosis according to a standard protocol. Ileum sections were sectioned at $5 \mu \mathrm{m}$. Images were captured at $200 \times$ via an Olympus BX43 microscope and CellSens Software package (Olympus America, Breinigsville, PA, USA). Quantitation of ileum fibrosis was assessed by two independent investigators quantifying percent area of Sirius red staining relative to percent area of ileum tissue section in Image J as has been previously described [51]. 5 randomized images per ileum section were used for the Image J analysis. 


\subsection{Ileum Tissue RNA Isolation and Quality Analysis}

Total ileum RNA was isolated using Trizol reagent (Thermo Fisher, Waltham, MA, USA) from murine ileal segments. This was followed by removal of any contaminating genomic DNA with DNase I (TURBO DNA-free kit, Thermo Fisher). RNA was further purified using the GeneJET RNA cleanup and concentration micro kit (Thermo Fisher). RNA integrity was determined by analysis on the Agilent Bioanalyzer 2100 (Agilent, Santa Clara, CA, USA) and only RNA samples with integrity values ranging from 7 to 9 were used for RNA-seq analysis.

\subsection{Intestinal Tissue RNA-Sequencing}

RNA sequencing was performed by the University of Louisville Center for Genetics in Molecular Medicine core facility using the TruSeq Stranded mRNA library preparation kit (part no. 20020594). The full RNASeq methodology has been reported previously [15].

\subsection{RNA-seq Bioinformatics, Statistical Analysis, and Data Visualization}

RNA-seq analysis was conducted by the NIH-funded Kentucky Biomedical Research Infrastructure Network Bioinformatics Core. The full details for this method have been published previously [15], but pertinent details are described below. The sequences were directly aligned to the Mus musculus reference genome assembly (mm10) using TopHat2 (version 2.0.13) [52] guided by Ensembl build 82 mouse transcripts. DEGs between experimental groups were identified using the Tuxedo suite of programs including cuffdiff2 (version 2.2.1) [53,54]. A $p$-value cutoff $\leq 0.05, q$-value cutoff $\leq 0.05$ with a fold change $(\mathrm{FC}) \geq 2$ was used to determine differential expression. DEGs that met this threshold were used for further analysis. Visualization of gene clusters and gene interactions for DEGs was conducted in Cytoscape [55]. Heatmap gradient coloration denotes $\log _{2}$ (FC) for the respective comparison and node size depicts relative connectivity from the Search Tool for the Retrieval of Interacting Genes/Proteins (STRING) database [56]. Similarly, Gene Ontology: Biological Processes (GO:BPs) and Kyoto Encyclopedia of Genes and Genomes (KEGG) Pathways that included the DEGs were conducted in Cytoscape [55]. Cytoscape analysis identified enriched GO:BPs (representative of DEGs increased), diminished GO:BPs (representative of DEGs decreased) for their respective comparisons. The top ten enriched and diminished GO:BPs were reported along with their associated false-discovery rate (FDR $<0.05)$. Similarly, STRING Analysis and gene clustering were conducted in Cytoscape followed by KEGG Pathway enrichment (FDR < 0.05). Gene clusters and their associated KEGG Pathway were used for DEGs increased and decreased for the respective comparisons. The GEO accession number for the RNAseq data reported in this paper is GSE133253.

\section{Conclusions}

The ileal transcriptional responses in an EtOH-acute systemic inflammation model were described in WT and fat-1 mice. Many transcriptional responses were similar between WT and fat-1 mice in response to EtOH + LPS vs. EtOH, but others were distinct. This study identified ileum APRIL signaling and IgA + B cell gene signatures as being positively upregulated by n-3 PUFA enrichment in this model. In addition, the expression of pro-restorative macrophage and $\gamma \delta \mathrm{T}$ cell genes was increased by n-3 PUFA enrichment in contrast to pro-fibrotic genes, which were decreased in fat- 1 mice. Reduced fibrosis was confirmed by Sirius red staining. Follow-up studies are required to further validate these findings and to determine the implications in alcohol and acute inflammation- mediated intestinal pathology in humans. These data demonstrate that n3-PUFAs can significantly modify the transcriptional response to EtOH + LPS, which may mitigate many of the deleterious effects on the gut and therefore may be a therapeutic strategy to treat intestinal damage. 
Supplementary Materials: The following are available online at https:/ / www.mdpi.com/1422-006 7/22/4/1582/s1.

Author Contributions: Listed below are the author contributions to this study. Conceptualization, I.A.K., D.R.W., J.E.H., J.B.W.; methodology, E.C.R., J.E.H.; software, E.C.R., J.E.H.; formal analysis, I.A.K., E.C.R., J.E.H., J.B.W., D.R.W., Y.L.S.; investigation, I.A.K., D.R.W., J.B.W., J.E.H.; resources, I.A.K., C.J.M., E.C.R.; data curation, E.C.R.; writing-original draft preparation, J.E.H., I.A.K.; writing-review and editing, J.E.H., J.B.W., D.R.W., E.C.R., C.J.M., I.A.K.; visualization, J.E.H., I.A.K., J.B.W.; supervision, I.A.K.; project administration, I.A.K.; funding acquisition, I.A.K., C.J.M., E.C.R., J.E.H., J.B.W. All authors have read and agreed to the published version of the manuscript.

Funding: The work presented in this study was supported by NIH grants T32ES011564 (J.E.H., J.B.W.), F32AA027950-01A1 (J.E.H.), R01 AA024102-01A1, F31AA028423-01A1 (J.B.W.) (I.A.K.), U01AA022489 (C.J.M.), 1U01AA021901-01 (C.J.M.), 1U01AA021893-01 (C.J.M.), R01AA023681 (C.J.M.), P20GM103436 (E.C.R.), P30GM106396, the Department of Veterans Affairs I01BX000350 (C.J.M.), and the Intramural Programs of the National Institute on Alcohol Abuse and Alcoholism. Research reported in this publication was supported by an Institutional Development Award (IDeA) from the National Institute of General Medical Sciences of the National Institutes of Health under grant number P20GM113226 (C.J.M.), and the National Institute on Alcohol Abuse and Alcoholism of the National Institutes of Health under Award Number P50AA024337 (C.J.M.). The content is solely the responsibility of the authors and does not necessarily represent the official views of the National Institutes of Health.

Institutional Review Board Statement: This study was conducted according to the guidelines of the University of Louisville Institutional Animal Care and Use Committee (IACUC). The IACUC protocol numbered 15423 was approved on 12 January 2016 by the IACUC ethics committee.

Data Availability Statement: Data are available in the publicly accessible GEO Data repository. Data can be found under the following accession number [GSE133253].

Acknowledgments: The authors thank Marion McClain for manuscript editing. We also wish to acknowledge Shubha Gosh-Dastidar for her contributions to this project.

Conflicts of Interest: The authors declare no conflict of interest.

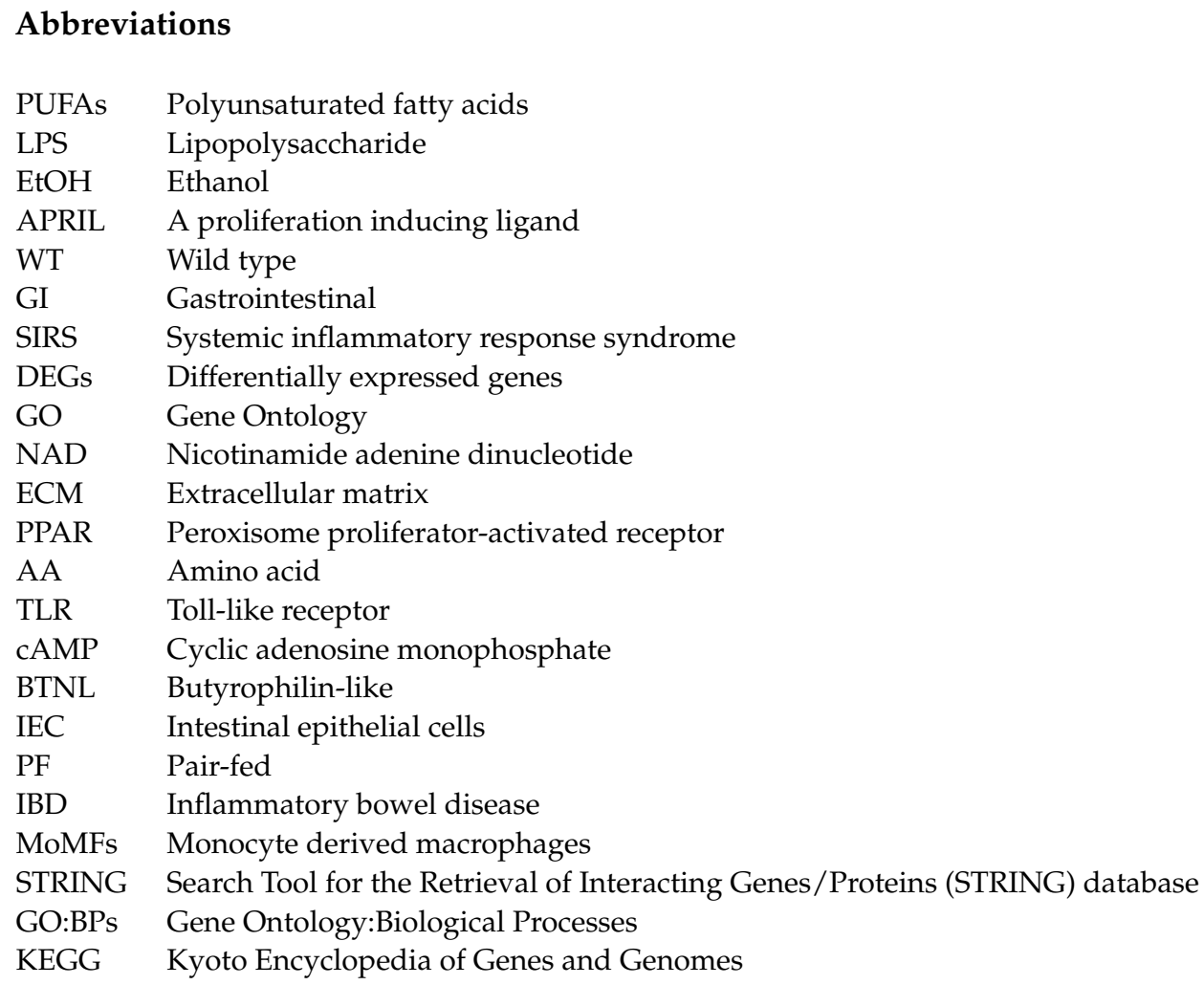




\section{References}

1. Peterson, L.W.; Artis, D. Intestinal epithelial cells: Regulators of barrier function and immune homeostasis. Nat. Rev. Immunol. 2014, 14, 141-153. [CrossRef]

2. Ungaro, F.; Rubbino, F.; Danese, S.; D'Alessio, S. Actors and Factors in the Resolution of Intestinal Inflammation: Lipid Mediators As a New Approach to Therapy in Inflammatory Bowel Diseases. Front. Immunol. 2017, 8, 1331. [CrossRef]

3. Costantini, L.; Molinari, R.; Farinon, B.; Merendino, N. Impact of Omega-3 Fatty Acids on the Gut Microbiota. Int. J. Mol. Sci. 2017, 18, 2645. [CrossRef]

4. Whiting, C.V.; Bland, P.W.; Tarlton, J.F. Dietary n-3 polyunsaturated fatty acids reduce disease and colonic proinflammatory cytokines in a mouse model of colitis. Inflamm. Bowel Dis. 2005, 11, 340-349. [CrossRef]

5. Kang, J.X.; Wang, J.; Wu, L.; Kang, Z.B. Transgenic mice: Fat-1 mice convert n-6 to n-3 fatty acids. Nature 2004, 427, 504. [CrossRef]

6. Bidu, C.; Escoula, Q.; Bellenger, S.; Spor, A.; Galan, M.; Geissler, A.; Bouchot, A.; Dardevet, D.; Morio, B.; Cani, P.D.; et al. The Transplantation of $\omega 3$ PUFA-Altered Gut Microbiota of fat-1 Mice to Wild-Type Littermates Prevents Obesity and Associated Metabolic Disorders. Diabetes 2018, 67, 1512-1523. [CrossRef]

7. Hudert, C.A.; Weylandt, K.H.; Lu, Y.; Wang, J.; Hong, S.; Dignass, A.; Serhan, C.N.; Kang, J.X. Transgenic mice rich in endogenous omega-3 fatty acids are protected from colitis. Proc. Natl. Acad. Sci. USA 2006, 103, 11276-11281. [CrossRef]

8. Ghosh, S.; Molcan, E.; DeCoffe, D.; Dai, C.; Gibson, D.L. Diets rich in n-6 PUFA induce intestinal microbial dysbiosis in aged mice. Br. J. Nutr. 2013, 110, 515-523. [CrossRef]

9. Kirpich, I.A.; Petrosino, J.; Ajami, N.; Feng, W.; Wang, Y.; Liu, Y.; Beier, J.I.; Barve, S.S.; Yin, X.; Wei, X.; et al. Saturated and Unsaturated Dietary Fats Differentially Modulate Ethanol-Induced Changes in Gut Microbiome and Metabolome in a Mouse Model of Alcoholic Liver Disease. Am. J. Pathol. 2016, 186, 765-776. [CrossRef]

10. Kirpich, I.A.; Feng, W.; Wang, Y.; Liu, Y.; Barker, D.F.; Barve, S.S.; McClain, C.J. The type of dietary fat modulates intestinal tight junction integrity, gut permeability, and hepatic toll-like receptor expression in a mouse model of alcoholic liver disease. Alcohol Clin. Exp. Res. 2012, 36, 835-846. [CrossRef]

11. Kirpich, I.A.; Feng, W.; Wang, Y.; Liu, Y.; Beier, J.I.; Arteel, G.E.; Falkner, K.C.; Barve, S.S.; McClain, C.J. Ethanol and dietary unsaturated fat (corn oil/linoleic acid enriched) cause intestinal inflammation and impaired intestinal barrier defense in mice chronically fed alcohol. Alcohol 2013, 47, 257-264. [CrossRef]

12. Yan, A.W.; Schnabl, B. Bacterial translocation and changes in the intestinal microbiome associated with alcoholic liver disease. World J. Hepatol. 2012, 4, 110-118. [CrossRef]

13. Haussner, F.; Chakraborty, S.; Halbgebauer, R.; Huber-Lang, M. Challenge to the Intestinal Mucosa During Sepsis. Front. Immunol. 2019, 10, 891. [CrossRef] [PubMed]

14. Rieder, F. The gut microbiome in intestinal fibrosis: Environmental protector or provocateur? Sci. Transl. Med. 2013, 5, 190ps10. [CrossRef] [PubMed]

15. Warner, D.R.; Warner, J.B.; Hardesty, J.E.; Song, Y.L.; King, T.N.; Kang, J.X.; Chen, C.Y.; Xie, S.; Yuan, F.; Prodhan, M.A.I.; et al. Decreased $\omega-6: \omega-3$ PUFA ratio attenuates ethanol-induced alterations in intestinal homeostasis, microbiota, and liver injury. J. Lipid Res. 2019, 60, 2034-2049. [CrossRef]

16. Hardesty, J.E.; Warner, J.B.; Song, Y.L.; Rouchka, E.C.; Chen, C.Y.; Kang, J.X.; McClain, C.J.; Warner, D.R.; Kirpich, I.A. Transcriptional signatures of the small intestinal mucosa in response to ethanol in transgenic mice rich in endogenous n3 fatty acids. Sci. Rep. 2020, 10, 19930. [CrossRef]

17. Di Marco Barros, R.; Roberts, N.A.; Dart, R.J.; Vantourout, P.; Jandke, A.; Nussbaumer, O.; Deban, L.; Cipolat, S.; Hart, R.; Iannitto, M.L.; et al. Epithelia Use Butyrophilin-like Molecules to Shape Organ-Specific $\gamma \delta$ T Cell Compartments. Cell 2016, 167, 203-218.e17. [CrossRef]

18. Willcox, C.R.; Vantourout, P.; Salim, M.; Zlatareva, I.; Melandri, D.; Zanardo, L.; George, R.; Kjaer, S.; Jeeves, M.; Mohammed, F.; et al. Butyrophilin-like 3 Directly Binds a Human $V \gamma 4(+)$ T Cell Receptor Using a Modality Distinct from Clonally-Restricted Antigen. Immunity 2019, 51, 813-825.e4. [CrossRef]

19. Na, Y.R.; Stakenborg, M.; Seok, S.H.; Matteoli, G. Macrophages in intestinal inflammation and resolution: A potential therapeutic target in IBD. Nat. Rev. Gastroenterol. Hepatol. 2019, 16, 531-543. [CrossRef]

20. Lycke, N.Y.; Bemark, M. The regulation of gut mucosal IgA B-cell responses: Recent developments. Mucosal Immunol. 2017, 10, 1361-1374. [CrossRef]

21. He, B.; Xu, W.; Santini, P.A.; Polydorides, A.D.; Chiu, A.; Estrella, J.; Shan, M.; Chadburn, A.; Villanacci, V.; Plebani, A.; et al. Intestinal bacteria trigger $\mathrm{T}$ cell-independent immunoglobulin $\mathrm{A}(2)$ class switching by inducing epithelial-cell secretion of the cytokine APRIL. Immunity 2007, 26, 812-826. [CrossRef]

22. Rieder, F.; Fiocchi, C. Intestinal fibrosis in IBD-a dynamic, multifactorial process. Nat. Rev. Gastroenterol. Hepatol. 2009, 6, $228-235$. [CrossRef]

23. Bode, C.; Bode, J.C. Effect of alcohol consumption on the gut. Best Pract. Res. Clin. Gastroenterol. 2003, 17, 575-592. [CrossRef]

24. Kirpich, I.A.; McClain, C.J.; Vatsalya, V.; Schwandt, M.; Phillips, M.; Falkner, K.C.; Zhang, L.; Harwell, C.; George, D.T.; Umhau, J.C. Liver Injury and Endotoxemia in Male and Female Alcohol-Dependent Individuals Admitted to an Alcohol Treatment Program. Alcohol Clin. Exp. Res. 2017, 41, 747-757. [CrossRef]

25. Moschen, A.R.; Adolph, T.E.; Gerner, R.R.; Wieser, V.; Tilg, H. Lipocalin-2: A Master Mediator of Intestinal and Metabolic Inflammation. Trends Endocrinol. Metab. 2017, 28, 388-397. [CrossRef] 
26. Dai, X.M.; Zong, X.H.; Sylvestre, V.; Stanley, E.R. Incomplete restoration of colony-stimulating factor 1 (CSF-1) function in CSF-1-deficient Csf1op/Csf1op mice by transgenic expression of cell surface CSF-1. Blood 2004, 103, 1114-1123. [CrossRef]

27. Matera, I.; Rusmini, M.; Guo, Y.; Lerone, M.; Li, J.; Zhang, J.; Di Duca, M.; Nozza, P.; Mosconi, M.; Pini Prato, A.; et al. Variants of the ACTG2 gene correlate with degree of severity and presence of megacystis in chronic intestinal pseudo-obstruction. Eur. J. Hum. Genet. 2016, 24, 1211-1215. [CrossRef]

28. Gonzalo, S.; Grasa, L.; Árruebo, M.P.; Plaza, M.; Murillo, M.D. Lipopolysaccharide-induced intestinal motility disturbances are mediated by c-Jun NH2-terminal kinases. Dig. Liver Dis. 2011, 43, 277-285. [CrossRef]

29. Puleston, J.; Cooper, M.; Murch, S.; Bid, K.; Makh, S.; Ashwood, P.; Bingham, A.H.; Green, H.; Moss, P.; Dhillon, A.; et al. A distinct subset of chemokines dominates the mucosal chemokine response in inflammatory bowel disease. Aliment. Pharm. Ther. 2005, 21, 109-120. [CrossRef]

30. Scarpa, M.; Kessler, S.; Sadler, T.; West, G.; Homer, C.; McDonald, C.; de la Motte, C.; Fiocchi, C.; Stylianou, E. The epithelial danger signal IL- $1 \alpha$ is a potent activator of fibroblasts and reactivator of intestinal inflammation. Am. J. Pathol. 2015, 185, 1624-1637. [CrossRef]

31. Shores, D.R.; Binion, D.G.; Freeman, B.A.; Baker, P.R. New insights into the role of fatty acids in the pathogenesis and resolution of inflammatory bowel disease. Inflamm. Bowel Dis. 2011, 17, 2192-2204. [CrossRef]

32. Jakobsson, T.; Vedin, L.L.; Hassan, T.; Venteclef, N.; Greco, D.; D'Amato, M.; Treuter, E.; Gustafsson, J.; Steffensen, K.R. The oxysterol receptor LXR $\beta$ protects against DSS- and TNBS-induced colitis in mice. Mucosal Immunol. 2014, 7, 1416-1428. [CrossRef]

33. Perochon, J.; Carroll, L.R.; Cordero, J.B. Wnt Signalling in Intestinal Stem Cells: Lessons from Mice and Flies. Genes 2018, 9 , 138. [CrossRef]

34. Kappeler, A.; Mueller, C. The role of activated cytotoxic T cells in inflammatory bowel disease. Histol. Histopathol. 2000, 15, 167-172.

35. Klepsch, V.; Moschen, A.R.; Tilg, H.; Baier, G.; Hermann-Kleiter, N. Nuclear Receptors Regulate Intestinal Inflammation in the Context of IBD. Front. Immunol. 2019, 10, 1070. [CrossRef]

36. Kaiko, G.E.; Chen, F.; Lai, C.W.; Chiang, I.L.; Perrigoue, J.; Stojmirović, A.; Li, K.; Muegge, B.D.; Jain, U.; VanDussen, K.L.; et al. PAI-1 augments mucosal damage in colitis. Sci. Transl. Med. 2019, 11, eaat0852. [CrossRef]

37. Mehta, J.; Lawson, D.; Saldeen, T.J. Reduction in plasminogen activator inhibitor-1 (PAI-1) with omega-3 polyunsaturated fatty acid (PUFA) intake. Am. Heart J. 1988, 116, 1201-1206. [CrossRef]

38. Nielsen, M.M.; Witherden, D.A.; Havran, W.L. $\gamma \delta$ T cells in homeostasis and host defence of epithelial barrier tissues. Nat. Rev. Immunol. 2017, 17, 733-745. [CrossRef]

39. Nicolaou, A.; Mauro, C.; Urquhart, P.; Marelli-Berg, F. Polyunsaturated Fatty Acid-derived lipid mediators and T cell function. Front. Immunol. 2014, 5, 75. [CrossRef]

40. Fan, Y.Y.; Spencer, T.E.; Wang, N.; Moyer, M.P.; Chapkin, R.S. Chemopreventive n-3 fatty acids activate RXRalpha in colonocytes. Carcinogenesis 2003, 24, 1541-1548. [CrossRef]

41. Triantafyllou, E.; Pop, O.T.; Possamai, L.A.; Wilhelm, A.; Liaskou, E.; Singanayagam, A.; Bernsmeier, C.; Khamri, W.; Petts, G.; Dargue, R.; et al. MerTK expressing hepatic macrophages promote the resolution of inflammation in acute liver failure. Gut 2018, 67, 333-347. [CrossRef]

42. Rothlin, C.V.; Leighton, J.A.; Ghosh, S. Tyro3, Axl, and Mertk receptor signaling in inflammatory bowel disease and colitisassociated cancer. Inflamm. Bowel Dis. 2014, 20, 1472-1480. [CrossRef]

43. Menckeberg, C.L.; Hol, J.; Simons-Oosterhuis, Y.; Raatgeep, H.R.; de Ruiter, L.F.; Lindenbergh-Kortleve, D.J.; Korteland-van Male, A.M.; El Aidy, S.; van Lierop, P.P.; Kleerebezem, M.; et al. Human buccal epithelium acquires microbial hyporesponsiveness at birth, a role for secretory leukocyte protease inhibitor. Gut 2015, 64, 884-893. [CrossRef]

44. Bunker, J.J.; Erickson, S.A.; Flynn, T.M.; Henry, C.; Koval, J.C.; Meisel, M.; Jabri, B.; Antonopoulos, D.A.; Wilson, P.C.; Bendelac, A. Natural polyreactive IgA antibodies coat the intestinal microbiota. Science 2017, 358, eaan6619. [CrossRef]

45. Corthésy, B. Multi-faceted functions of secretory IgA at mucosal surfaces. Front. Immunol. 2013, 4, 185. [CrossRef]

46. Teague, H.; Harris, M.; Fenton, J.; Lallemand, P.; Shewchuk, B.M.; Shaikh, S.R. Eicosapentaenoic and docosahexaenoic acid ethyl esters differentially enhance B-cell activity in murine obesity. J. Lipid Res. 2014, 55, 1420-1433. [CrossRef]

47. López, M.C. Chronic alcohol consumption regulates the expression of poly immunoglobulin receptor (pIgR) and secretory IgA in the gut. Toxicol. Appl. Pharmacol. 2017, 333, 84-91. [CrossRef]

48. Inamine, T.; Yang, A.M.; Wang, L.; Lee, K.C.; Llorente, C.; Schnabl, B. Genetic Loss of Immunoglobulin A Does Not Influence Development of Alcoholic Steatohepatitis in Mice. Alcohol Clin. Exp. Res. 2016, 40, 2604-2613. [CrossRef]

49. Dammeier, J.; Brauchle, M.; Falk, W.; Grotendorst, G.R.; Werner, S. Connective tissue growth factor: A novel regulator of mucosal repair and fibrosis in inflammatory bowel disease? Int. J. Biochem. Cell Biol. 1998, 30, 909-922. [CrossRef]

50. Zhang, K.; Chang, Y.; Shi, Z.; Han, X.; Han, Y.; Yao, Q.; Hu, Z.; Cui, H.; Zheng, L.; Han, T.; et al. w-3 PUFAs ameliorate liver fibrosis and inhibit hepatic stellate cells proliferation and activation by promoting YAP/TAZ degradation. Sci. Rep. 2016, 6, 30029. [CrossRef]

51. Li, X.H.; Fang, Z.N.; Guan, T.M.; Lin, J.J.; Sun, C.H.; Huang, S.Y.; Mao, R.; Lu, B.L.; Cao, Q.H.; Feng, S.T.; et al. A novel collagen area fraction index to quantitatively assess bowel fibrosis in patients with Crohn's disease. BMC Gastroenterol. 2019, 19, 180. [CrossRef] 
52. Kim, D.; Pertea, G.; Trapnell, C.; Pimentel, H.; Kelley, R.; Salzberg, S.L. TopHat2: Accurate alignment of transcriptomes in the presence of insertions, deletions and gene fusions. Genome Biol. 2013, 14, R36. [CrossRef] [PubMed]

53. Trapnell, C.; Hendrickson, D.G.; Sauvageau, M.; Goff, L.; Rinn, J.L.; Pachter, L. Differential analysis of gene regulation at transcript resolution with RNA-seq. Nat. Biotechnol. 2013, 31, 46-53. [CrossRef] [PubMed]

54. Trapnell, C.; Roberts, A.; Goff, L.; Pertea, G.; Kim, D.; Kelley, D.R.; Pimentel, H.; Salzberg, S.L.; Rinn, J.L.; Pachter, L. Differential gene and transcript expression analysis of RNA-seq experiments with TopHat and Cufflinks. Nat. Protoc. 2012, 7, 562-578. [CrossRef] [PubMed]

55. Shannon, P.; Markiel, A.; Ozier, O.; Baliga, N.S.; Wang, J.T.; Ramage, D.; Amin, N.; Schwikowski, B.; Ideker, T. Cytoscape: A software environment for integrated models of biomolecular interaction networks. Genome Res. 2003, 13, 2498-2504. [CrossRef] [PubMed]

56. Szklarczyk, D.; Gable, A.L.; Lyon, D.; Junge, A.; Wyder, S.; Huerta-Cepas, J.; Simonovic, M.; Doncheva, N.T.; Morris, J.H.; Bork, P.; et al. STRING v11: Protein-protein association networks with increased coverage, supporting functional discovery in genome-wide experimental datasets. Nucleic Acids Res. 2019, 47, D607-D613. [CrossRef] 